\title{
On the Uncertainty Principle
}

\author{
Mesut KAVAK*
}

\begin{abstract}
Analysis of the laws which form, direct universe and of the interacting elements in the interactions emerging by these laws. Forming the theoretical, philosophical infrastructure of the some physical concepts and phenomena such as kinetic energy, uncertainty [1], length contraction [2], relative energy transformations, gravity, time and light speed to understand universe better manner as well as possible. Almost any physical subject takes us easily to the same point by visiting the other subjects because of the creation type of matter as there is no alternative. Every mathematical equation is a production of a thinking; so it does not have to be right always as it may has different meanings for different minds and because of wrong thinking, assembly elements even if it may has a certain information sometimes or usually. If our portion is, our portion is as we took some pickaxes and shovels, starting for science mining as the below to understand universe better manner, if it is possible especially by strong evidences by thinking first as simple as possible. Only one pencil and one paper are enough. At this situation, the biggest problem is to be one of them exists and the other does not of them.
\end{abstract}

\section{Introduction}

Work potential exactly means total energy, and it causes the physical facts which have not been there somewhere yet or have been enriched our scientific knowledge for centuries. It is the only basic function which these facts can make sense on it.

When uncertainty warned, total energy must be limited and at last fixed; so the total energy which is formed in this way is not able to give matter infinite work ability. Uncertainty shows itself for mechanical machines beside some usual human characteristics which can be reproduced such as talking, thinking, walking and sex. The works like all of these works can only occur in a time interval together with a motion which repeats itself at a frequency; but they are not at everywhere at the same time untimely manner. Uncertain energy increases or decreases between absoluteness and absolute $a b-$ sence; but it never disappears. This fact always protects and verifies itself in every combination of any action.

Matter which is not infinite sustains its existence as waves over the infinite, and it must be a part of God by the reason of must be worked and has been working of an infinite, before all of the workers instead of the workers as required by uncertainty. God does this creation work, and his work can only occur by some potential differences, and as this differences cause gravity, it is not possible to find another energy resource except gravity; because God does this work against recovery of space every second and actually in every small periods of time. As kinetic energy is equal to work done, matter's all the work is equal to the work done by gravity.

Universe is space itself, and particles are waves which emerge on the space as second waves, and it has an amount of disorder equal to its total energy every second that this disorder is recovered by forcing each second as work done is equal to total energy and thus equal to disorder; thus even if particles are included to the total energy of universe, they lose their energies during this loss of universe at an amount every second and want to be space without waves again; because they emerge over a space that wants to be fixed, and as no work can only be done untimely manner, a disorder period occurs and excludes particles in the other name space waves from the space. This is the same event with it, that if you stretch a curtain by hand which was already stretched, you feel a resistance. Like in this event as particles have no other external energy, they lose their energies by some periods, external frictional and gravitational losses are not included as well. Even the space without waves wants to be fixed as well, God always forces it to emerge of it during its emergence even during the smallest slice of time; thus even the day when there would be no particle photon is included as well in space, space itself cannot be fixed if the forcing is not removed. Another alternative is not possible in the point of the formation type; thus disorder is an absolute law.

God and creatures have no alternative in the point of creating and to be created; but there are infinite numbers of different creatures over the single existence type. It does not differ for matter, that it is created or it works during the creation or during another work which is after the creation. Mater uses the same space at the same time during every existing action. It does work equal to its total energy because of its existence even if it is not used, and it is able to work equal to its total energy because of this availability. This potential is a great potential for making matter worked by using its own energy every second without external energy. This energy can be transformed into force easily as well as energy and mass thus force is not independent of each other. By this way, we can easily establish some main technological vital structures of mankind without any other main instruments needed.

\section{Theoretical Analysis of Kinetic Energy}

Velocity is the rate of change of position with respect to a frame of reference. It is not independent of distance, energy, mass and time just as distance, energy, mass and time are not independent of velocity; because they are natural consequences of each other.

A matter does work even if it does work relatively to us in outer space observational area by interacting another space elements by using its own energy without external energy source or when made it worked by man-made forcing with external energy, and work can only be done as much as energy existence as kinetic energy is equal to work done. Energy is not independent of velocity, mass, distance and time; thus for creation of a mass, a motion; thus time, distance and velocity must occur without any priority to each other as mass is only possible with existence of energy, and energy is not independent of velocity. This existence condition can be maintained as

A motionless mass cannot exist.

Proposition 1

A motion occurs together with a work; thus a mass cannot get the same mass magnitude at the smaller periods of time; because the energy expended for a work which is done to create a mass closes to infinite. Work done is equal to kinetic energy; thus closing to infinite means, that even the smallest 
mass part of universe gains a potential which is infinite work ability; thus a mass always must get smaller mass magnitude at the smaller periods of time. This means, that work cannot be done untimely manner; thus mass cannot be formed without time, and it cannot be absolute. This existence condition can be maintained as

Matter is not absolute.

\section{Proposition 2}

Absolute absence of matter means, that matter has a frequency as it cannot exist everywhere untimely manner. This existence condition of matter means, that matter can only exist in some time intervals and it does not exist at the same periods of time; thus it does work in some periods and does not work in the same periods. This condition is the condition of inertia, and as it can be seen inertia means, that during the work matter stores energy in the times when matter does not do work to do work; thus this existence condition can be maintained as

Matter stores energy.

\section{Proposition 3}

As matter is not absolute, constant speed movement can only be created as it cannot be absolute, and is relative. Energy can exist in three main conditions which are absolute energy, caused by the acceleration from zero point and caused by the acceleration which is because of a deceleration from any magnitude existed energy the infinite energy is included as well. As matter has no infinite work ability, matter cannot emerge by the acceleration from zero point because of the previous energy need.

Distance taken is dependent of classic mechanics equations that they are velocity and acceleration equations; thus if it is assumed that $d$ is the distance taken, then they are dependent of $d=v t$ and $d=a t^{2}$ equation which can be expressed by the other expressions as $y=x$ and $y=x^{2}$ functions according to time. As the intersection point of the functions is $x=1$ second, velocity and acceleration increase to the same value at the end of 1 second. Action being the same, the road proceeded during the action performed for the matter which starts to occur after the moment of $t=0$ second must be the same road; thus this to be at the same road in $1 \geq t \geq 0$ time interval condition means that the acceleration decreases from an infinite value as a part of infinite. The existence condition can be maintained as

Matter decelerates from a timeless thus an absolute energy.

\section{Proposition 4}

Deceleration cannot emerge from nothingness because of the previous energy need, and matter can only be created by forcing every second as matter is a virtual part of the infinite as you can neither detract anything from, nor add anything to infinite, since everything is an element of the infinite; thus it can be maintained as

Matter is absolute inert.

\section{Proposition 5}

This state of absolute inertia means, that matter basically does not have a potential to perform an action. The matter emerges as the result of this action cannot be absolute, and it can only be used as much as amount of its existence. This existence condition can be maintained as

Matter can perform action as much as its total energy. The lowest energy zone determines the efficiency of a system.

\section{Proposition 6}

This theoretically means an energy potential with a magnitude of $m v^{2}$ which has "Joule per second" that can be shorten as $(J s)$ unite for a creation velocity of $v$. For example in a simple machine system which provides force efficiency, if you enlarge the magnets gradually for the fixed force efficiency distance which is the other hand of the system, even if the magnetic flux change number is the same, the taken road for each change must be decrease at the amount of the enlarged magnets for the same force; thus the work done decreases even if the generated energy is the same or increases, and finally even electric can be produced without external energy, that means zero external work done if you use big enough magnets. In a high magnetic field, self-inflicted electron flow means electricity can be detected beyond the same order of spins because of electrons' movements which occur at different distances around their own atoms; because even if the high magnetic field is fixed, motionless electron's movement in this magnetic field around own atom can be counted as magnetic flux change. Also for example we can use instead of the lever two generators/motors to generate electricity. Let us assume, that one of them is $5 \mathrm{kw}$ at 100 turns, and the other one is $1 \mathrm{kw}$ at 500 turns. If you run the $1 \mathrm{kw}$ with an external electricity and then use $5 \mathrm{x}$ radius gear on the $5 \mathrm{kw}$ for $1 \mathrm{kw}$, you can turn the $5 \mathrm{kw}$. After that the $5 \mathrm{kw}$ can feed the $1 \mathrm{kw}$ by $1 / 5$ of its energy. Only the magnet's weight effects the condition. Self-feeding systems are possible with or without magnets by many different techniques with some auto vacuum layers against sound. Also it is possible for fusion energy; because as stated the above, a motionless mass cannot exist; thus you can't only calculate the given energy. Answer of matter's own velocityinduced energy in the other name extra creation movement reaction is important and is included to the calculations with the given energy. This is like the condition of a gearwheel turns at a fixed speed. If you give a handle to the system, the gear will hit the handle forever and will work, and the gear's energy is in the God's hands. Also it is valid for electromagnetism. Electrons and nucleus use their own energies in changing magnetic flux, field. This is not like all the usual works of classic mechanics, for example is not as hydraulics. They are only similar from the outside. We can force matter by some incitements to work in the name of us by using some amounts of its own $m v^{2}(J S)$ energy. Also we can use vacuum for induction coils or use superconductors directly.

Absolute absence of matter means uncertainty. Magnitudes of energy and mass are less in the smaller periods of time based on the reference time of absolute upper limit of 1 second. This means, that matter is more uncertain in the smaller periods of time. Uncertainty increases or decreases between absoluteness and absolute absence as matter is possible with an existent energy; but it never disappears. Heisenberg's Uncertainty Principle [1] basically means this. Matter is constantly uncertain for each dimension; because an uncertain emergence involves an uncertain matter.

Unlimited energy cannot be generated with a limited velocity as required by uncertainty since velocity is possible with an action; thus universe can have neither an infinite energy nor an infinite volume for existence of formation of visible motions and different masses. This existence condition can be maintained as

Universe does not have infinite energy and infinite volume.

\section{Proposition 7}

If total energy is limited, then velocity must be limited, and fixed at the end; thus different mass magnitudes for a fixed ve- 
locity are only possible with circular motion as different mass magnitudes means different displacements and consequently mean different velocities; so it can be collected as

Matter can only perform circular motion during the creation.

\section{Proposition 8}

If $m_{b}$ is the assumed basic mass when uncertainty warns, and $m_{d}$ is the mass detected at the end of a $t_{r}$ reference time; by the defined elements, emergence of a mass can be expressed as

$$
m_{d}=m_{b} \int_{0}^{t_{r}} d_{t_{r}}
$$

The same $m_{d}$ mass has to get different magnitudes for any time between $\left[0, m_{d}\right]$ and "Kilograms per second" unit that can be shorten as $(K g s)$ unit for 1 second.

$$
v_{f}=m_{d} / m_{b} t_{r}
$$

(2) is the change of mass for a formation velocity of $v_{f}$, and it is equal to some variable values according to emergence values. This existence condition can be maintained as

Mass is a varying effect which is detected within displacement time.

\section{Proposition 9}

As displacement of the mass which moves in outer space observational area after the emerging increases for the reference time of absolute upper limit of 1 second, also its mass magnitude must increase absolutely; because a motion in outer space cannot be independent of emergence just as emergence cannot be; thus the existence condition can be maintained as

Masses of the moving objects increase.

\section{Proposition 10}

For $m_{d}(K g s)$ mass which had been accelerated after it emerged, as reference time of $t_{r}$ is 1 second and $m_{s}$ is an outer space motion mass, it can be written

$$
v=m_{S} t / m_{d} t_{r}
$$

and $m_{d}(K g s)$ mass is perceived as an $m_{s}(K g s)$ mass according to the acceleration time $t$ by the reason of inertia. There is no other mass. During the acceleration work, $m_{d}(K g s)$ can be assumed as the basic mass. The velocity here is equal to the velocity of the mass which moves in observational outer space if the mass moves. If the mass does not move, this velocity is already the velocity of the change of the mass in time as a detected mass. For $m_{d}=m$ to be more clear of it, this condition can be maintained as

$$
f t=m v
$$

as $m_{s}(K g s)$ mass is going to get its standard mass magnitude if it slows down; thus this mass can be assumed as a force as it is variable, temporary. This equation is the momentum equation, and momentum means the mass relatively to time. It is still mass in short.

An emptiness by the reason of absolute energy deficiency is not possible because of deceleration from an absolute energy; thus space allows matter to move and thus emerge at the every point of space. This means, that all masses are a single mass. When turned back to the beginning of time, then it becomes more understandable. It can be said, that there is an emergence by turns. This condition brings about emergence move around matter itself as required by the single universe mass; but also brings about a perfect entanglement as relativity warned by the reason of masses in an infinite number. The condition can be maintained as

All masses are accepted as a single mass due to entanglement, and universe occurs with time differences between different masses.

\section{Proposition 11}

It can be assumed, that there are infinite number of masses instead of single mass by having the same time difference again. The time difference can be used theoretically for determination of the basic emergence age; because the time difference can be taken to over threshold value as matter stores energy in theory. For example, we can find out the different one in a space stuff group by this way. Nothing is perfect because of the time difference; thus there is no symmetry in the universe, and also will not be in future as well. At this point, we can assume that there is a perfect chaos or perfect order. These two comments are the same.

Work can only be done in a time interval by the reason of uncertainty; thus a work is always done against a resistance for energies which have any magnitude God is included as well; thus by the reason of conservation of the energy which is limited due to the fixed and limited light speed, the temperature and the heat differences which emerge because of work done is always together with work; thus the condition can be maintained as

There is no kind of energy which cannot turn into mechanical energy.

\section{Proposition 12}

The condition becomes more understandable by the reason of the matter which has $e / c^{2}(K g s)$ mass; thus temperature must be matter as everything with $e / c^{2}(K g s)$ mass. If this is the fact, then universe must be the heat which is equal to its total energy, and the heat becomes more certain or uncertain according to works done by using from the total energy of universe. Emergence velocity must be limited even if light speed is not an emergence velocity by the reason of limited uncertain universe, and must be fixed at last; thus universe cannot be unstable and cannot have an amount of disorder; because nothing loses from the total energy of universe. The things which are unstable are masses at the focal points of universe; thus universe can turn into a swimming pool without any wave by the reason of mass in another saying particle loss in the focal points of universe. Right this point, also it can be said that total of the universe has a disorder amount which is equal to its total energy since always gets lost in each small time interval of small time interval, that the work done is done against this.

An absolute action threshold value for any action performed in works is not possible as the reason of one by one emergence as required by uncertainty. This means, that also each existent mass particle has an infinite number of mass particle; thus two different masses never intersect at the same mass magnitude. This means that it is not possible a flexible collision in universe and information is preserved forever as any certain mutual vector annihilation will not occur; thus the condition can be summarized as

Flexible collision is not possible in universe; thus information is preserved forever.

\section{Proposition 13}

Existence of flexible collisions is only assumed as the amount is too small. Information shrinks forever; but it never disappears. As to be absolute afterwards is not possible as the 
reason of the requirement of a timeless worker before which is told about it the above, also it is not possible to be lost because of the requirement of to be informed by the infinite. The infinite has to know all the infinite information untimely manner.

As universe has an amount of disorder in the focal points, also constant speed movement is not possible as well in universe because of this disorder; because matter cannot protect its energy thus its speed even during acceleration or when there is no external force to cause an acceleration thus during slowing at an amount even if the amount is too small. If we assume, that the total of universe has no disorder, as there are infinite number of masses and as universe is created by a forcing each second smaller periods of time are included as well, actually it can be said that all the universe has an amount of disorder which is equal to its total energy each second, and there is a regeneration at this point. As universe is created in infinite number of time and mass parts in 1 second, an infinite difference occurs for each infinite small part of constant movement for speed as slowing, and then it is suddenly recovered by God. Finally it seems as constant speed movement and is really constant speed movement as there is no alternative; but as it was said, in theory it is not possible absolutely as required by preservation of energy and information even if there is no slowing for constant speed of $c$ for the total of universe or the focal points of universe besides outer space movement relative to eye, that is in our dimension and as our usual works.

Information and matter must have the same building blocks as the requirement of to be information is having energy. All of the information must be at the same place because of entanglement. Because of the requirement of slowing down from the infinite, matter must exist as spatial waves on the infinite. The condition can be collected as

Matter is wave.

\section{Proposition 14}

Recovery of information is possible for any information type; but during the process, the information which is searched for and any information become the same at a point relatively for us but does not for the infinite.

As heat is single, also velocity is single for matter because of uncertainty and entanglement. Matter has been doing the same motion for the visible outer space motion which is different than the creation and the creation motion; so these two motions are accepted and emerge together, and the condition can be collected as

Outer space and creation motions are accepted and emerge together. Matter uses the same space during any act.

\section{Proposition 15}

During motions, because of creation at the fixed light speed, the condition can be collected as

Light speed is the highest speed, and the lower is not possible as well.

\section{Proposition 16}

The condition does not allow kinetic energy to be like (5) as the below

$$
w=\int_{0}^{v} v d_{m v}
$$

; because the change amount of a mass at low speeds can be assumed as zero, and the speed always must be fixed and the light speed; thus these two conditions do not allow for integration separately even if actually they are natural results for each other. On the momentum equation stated the above as (4), kinetic energy must be (6) as the below

$$
f x=m v^{2}
$$

for $\mathrm{x}=\mathrm{vt}$. It is not different than a simple multiplication. The requirement for $m v^{2} / 2$ is the same force magnitude even in the smaller periods of time. The first and the assumed infinite small work must be $f t / s=m_{0} v_{1} / s$. The second work which is the closest to the first one is $f t / s=\left(m_{0}+2 m_{0}\right) v_{2} / s$ as magnitudes of masses increase as much as used reference time but the smallest slice of time being the same. If the final velocity $v_{f}$ is taken from the equation for the other works, then it will be

$$
v_{f}=\sum_{n=1}^{\infty} \frac{2 f t}{m_{0} n(n+1)}
$$

Because of $\sum_{n=1}^{\infty} \frac{1}{n(n+1)}=1$, it will be

$$
f t=m v / 2
$$

; then $\mathrm{v}=2 \mathrm{ft} / \mathrm{m}, \mathrm{f}=\mathrm{mv} / 2 \mathrm{t}$ and since work done is $f x=$ $(m v / 2 t)\left(2 f t^{2} / m\right)=f t v$, for $\mathrm{ft}=\mathrm{mv} / 2$ equation, it will be

$$
f x=m v^{2} / 2
$$

and it is kinetic energy as work done is equal to kinetic energy. Also equation $F=m a$ must be $F=m a / 2$ for the same rule. The condition is only possible for a single condition, if work done always has the same magnitude in the smaller periods of time. There is no formulaic qualify.

For the motions which have no initial velocity, if $x=v t$ and $v=a t$ then if equation $t=v / a$ is put on its own place on $x=v t$, the timeless velocity equation dances after this as

$$
v^{2}=a x
$$

$v^{2}=2 a x$ is wrong as well.

For the motions with initial velocity, Torricelli equation $v_{f}^{2}=v_{i}^{2}+2 a x$ gives wrong results. It does not differ, that you do a work as one-piece without hand taking with a $P=$ $F t$ as already this work is done one by one as required by uncertainty or by parts which are $p=f_{x} t_{y}$ parts; so if $v_{i}$ is the initial velocity, $v_{g}$ is the gained velocity different than the first velocity, $v_{f}$ is the final velocity, then it must be $v_{f}=v_{i}+v_{g}$ and $v_{g}=a_{g} t$. If they are the assembly elements, then for $v_{f}^{2}=\left(v_{i}+a_{g} t\right)^{2}$, it will be

$$
v_{f}^{2}=v_{i}^{2}+2 v_{i} a_{g} t+a_{g}^{2} t^{2}
$$

If the total displacement is $x_{f}, x_{i}$ is the gained road during $t$ time by the initial velocity, $x_{g}$ is the gained road by the gained velocity which is different than the first velocity during $t$ time, then it must be $x_{f}=x_{i}+x_{g}, x_{g}=a_{g} t^{2}$ and thus must be $t^{2}=\left(x_{f}-x_{i}\right) / a_{g}$. If the time is put on the place on (11), then it will be $v_{f}^{2}=v_{i}^{2}+2 v_{i} a_{g} t+a_{g} x_{f}-a_{g} x_{i}$. For $v_{i} t=x_{i}$, it will be $2 v_{i} a_{g} t=2 x_{i} a_{g}$, and the timeless velocity equation which has an initial velocity will appear as

$$
\begin{aligned}
& v_{f}^{2}=v_{i}^{2}+a_{g}\left(x_{i}+x_{f}\right) \\
& v_{f}^{2}=v_{i}^{2}+a_{g}\left(2 x_{i}+x_{g}\right)
\end{aligned}
$$

On these information, kinetic energy will be

$$
m\left(v_{i}^{2}+a_{g}\left(2 x_{i}+x_{g}\right)\right)
$$


or

$$
m\left(v_{i}^{2}+a_{g}\left(x_{i}+x_{f}\right)\right)
$$

Being $g$ is the gravitational acceleration when mass and gravitational force changes are omitted in a small $[0-x]$ interval, it is $m v^{2}=\max$ if both of the sides are multiplied with $m$ over $v^{2}=a x$, and then gravitational potential energy is going to be

$$
e_{p}=m g x
$$

\section{Relative Kinetic Energy}

When relativity warned, matter can exist in two main conditions for motions, that they are static and moving condition. Both of these works can be done as much as total kinetic energy; then the kinetic energy which is last and named as moving total kinetic energy must be equal to the kinetic energy which is gained by a visible outer space movement plus static total kinetic energy. This can be expressed as

$$
m_{0} c^{2}+m v^{2}=m c^{2}
$$

The momentum and the kinetic energy equations which were derived the above as (4) and (6) change by a rule, and the rule is determined by this energy transformation equation (17). For the light speed of $c$, the equation dances after this as

$$
m=\frac{m_{0}}{1-\frac{v^{2}}{c^{2}}}
$$

This mass is the mass which had been accelerated and is moving at a speed of $v$. For acceleration work, if $p_{0}=m_{0} c$ and $v=f t / m_{0}$ then for $\int m d_{v}$ it will be

$$
m=p_{0} \tanh ^{-1}\left(\frac{f t}{p_{0}}\right)
$$

The total energy will change during movements; thus the condition can be maintained as

Mass and energy are not preserved in the focal points of universe; but for the total of universe, they are preserved.

\section{Proposition 17}

and Proposition 17 is not the same thing with preservation of energy of work done. It means that energy of a particle is not fixed, and is very dynamic. Even we use matter's own total energy for work without an external energy, it does not mean work done energy is not preserved, that work done energy is always preserved.

As you can see on (18) or (19) the limit is $c$ for $v=f t / m_{0}$ equation; but actually work can be done by any big multiples of $c$ for $\mathrm{ft} / \mathrm{m}_{0}$ equation and $\mathrm{ft} / \mathrm{m}_{0}>c$ status. As math has no mind, we must re-determine the condition. For (19.1) the below,

$$
m_{n+1}=p_{n} \tanh ^{-1}\left(\frac{\left(f t / p_{0}\right)-\left\lfloor f t / p_{0}\right\rfloor}{p_{n}}\right)
$$

it will be (19.2) for $p_{n}=m_{n} c$ as

$$
m=\sum_{n=0}^{\left\lfloor f t / p_{0}\right\rfloor} m_{n+1}
$$

for the work's whole value as there is no difference for a onepiece work or work in pieces. For $\mathrm{ft} / \mathrm{m}_{o}<c$ status, (19) can be used. The multiplication of $f t$ does not change even if the mass or the energy on the momentum equation changes by any rule; thus also the $m v$ multiplication never changes as well; so the speed has been reached can be taken from $f t$, and the speed has been reached will be $f t / m$ that $m$ is (19.2) or (19) here, is not $m_{0}$. If the force moves together with the mass, it must be done a relative calculation for the force as well. A calculation as the above as (18), (19), (19.2) is only valid for particle colliders; because the force does not move with the mass here.

Everything is an element of infinite; thus you can neither detract anything from, nor add anything to infinite. If a work is not performed at the infinite speed untimely manner, all the composed information will remain as virtual due to absolute absence; thus matter will appear and disappear with a frequency as a part of infinite. This is only possible by circular motion since information is an element of infinite. The main reason for a circular motion must be this different than observational different sizes of masses. A space object experiences constant potential difference during formation because of the time differences. When wave starts to move in one direction, the formed place of wave goes to disappearance. At this time, wave moves to the newly formed space. This movement must take place in all interactions of universe due to entanglement as well apart from the basic creation even if the basic creation and even electrons are included as well. Mercury, although it is not the only reason still is a good example and is necessary for all orbiting objects the world is included as well. One day, the world's spin must relatively be rotated and then the sun must rise from the west, and the world's movement which is like an endless retro movement will be understood, and it will be seen with naked eye. By looking at the information, it could be stated as

Matter does spiral circular motion.

\section{Proposition 18}

The wave causes 3 dimensions, and it moves in 2 dimensions in total due to the helical movement; thus the wave must be mathematically one-dimensional; then it could be stated as

The mass density which is perceived is on the surface because of the wave. Matter forms a 3dimension sphere with the helical circular motion. The total path proceeded each second during this process is equal to the path proceeded around a 2-dimension circle for the radius of the sphere it forms.

\section{Proposition 19}

Another effect of this potential difference is in Lorentz contraction [2]; because during movements, uncertainty decreases and mass increases as stated the above as masses of the moving objects increase; thus existence increases in an unit of time and it reduces the deviation for each round during the spiral circular motion of mass; in that case it could be stated as

Matter experiences length contraction during its movement in outer space because of change in uncertainty.

\section{Proposition 20}

The length contraction can only occur in one direction because of the resultant direction of movement. This is the nature of all movements; because a motion can only occur in one direction as and for a resultant in space. This means that a sphere is transformed into an ellipsoid; thus when static density is $d_{0}$ and moving density is $d$, it will be

$$
d=\frac{d_{0}}{\left(1-\frac{v^{2}}{c^{2}}\right)^{2}}
$$


because of $d=\left(m_{0} /\left(1-\left(v^{2} / c^{2}\right)\right)\right) /\left((4 / 3) \pi r^{2} r\left(1-\left(v^{2} / c^{2}\right)\right)\right.$. For a $m_{0}$ magnitude object, the length contraction is as

$$
L=L_{0}\left(1-\frac{v^{2}}{c^{2}}\right)
$$

and these results are for the matters which had been accelerated and are moving at a speed of $v$. For acceleration work, the calculations must be done over $\int d d_{v}$ and $\int L d_{v}$.

These information contradict with Michelson-Morley experiment [3] in entanglement; because the speed of light is the creation speed and it is fixed due to the limited universe; so it is independent of aether, free space for the status of this experiment. This case is the same for any moving matter in any density. The rays of moving objects are at a constant speed, only their energies are vary. When the length contraction occurs, a number of physical properties change such as electrical and thermal conductivity, total energy of mass and heat capacity. Also we can turn unmanned space vehicles against length contraction around themselves each second at the amount of the length contraction if it becomes a problem for vehicle health for more speed, and this has a poor limit because of centrifugal force. Additionally, maybe the earth is the center of the universe, and the universe turns around the earth. This is like a retro movement if this is the condition. We can test it by length contraction. If we send a space vehicle which goes in the reverse direction of the earth's space movement direction then the vehicle first must experience a volume increase and mass decrease, second a volume decrease and mass increase. This says that the earth moves. Otherwise the vehicle directly experiences a mass increase and volume decrease. Also electric conductivity can be used based on length contraction.

\section{Formation of Matter}

Photon is the basic formation particle which is the mass moves at the speed of light due to the circular motion, and it must do a continuous circular motion while forming a particle or moving freely in space that during linear motion in space freely, as it does a helical motion which is assumed as creation circular motion, additionally it may do a second helical or low helical high circular motion with a small derivation in time at the same time with creation helical motion as an outer space helix. The distance taken every second in a particle or empty space must be $r=c / 2 \pi$ for $2 \pi r=c t$ and $t=1$ second. During the work, photon acts like a particle when $r=c / 2 \pi$ radius is counted as free circulation radius, and the photon has the same values as its formed ones for 1 second in each work. The area covered must be

$$
A=c^{2} / 4 \pi\left(m^{2}\right)
$$

based on $\pi r^{2}$. For $e(J s)$ as it emerges within 1 second, energy density in this area is

$$
d_{e}=4 \pi e / c^{2}\left(\mathrm{~J} s / \mathrm{m}^{2}\right)
$$

and it is assumed, that $e$ energy provides this equitation for $1 \mathrm{~m}^{2}$. The energy generated in 1 dimension along 1 meter for 1 second is $\sqrt{d_{e}}(J s)$; thus it will be

$$
e=4 \pi / c^{4}(J s)
$$

based on $e=\sqrt{d_{e}} / c(J s)$ for each distance taken at the speed of light of $c$ each second. This could be the real required Planck constant. This is the total energy of the basic creation wave named as photon which has 1 Hertz frequency, and it occurs along 1 second in 1 dimension. The mass creates this energy is

$$
m_{p h}=4 \pi / c^{6}(K g s)
$$

based on $e / c^{2}(K g s)$, and this is the photon mass which can be shorten as $m_{p h}$. This is the mass which can only be formed along 1 second; thus when it collides with an object, the total mass effect occurs at the end of 1 second; thus wave can only be a string. The force which causes this mass will be $f=$ $m a=m v$ for 1 second; thus it must be

$$
f=4 \pi / c^{5}(K g s)
$$

The spirit of God was hovering over the waters, and God said that let there be light, and there was light. God saw that the light was good, and he separated the light from the darkness.

\section{Genesis/The Beginning}

verses talk about it. Free photon vibrates all of the space in 3 dimensions according to the helical frequency with 1 dimensional helical motion. By this way, it either travels to far places or becomes more apparent from far places of course according to its energy as well, and also photon itself is a sound and particles have static, natural sound as well since assumed the smallest friction is because of mass. Photon does not have to come directly to eye because of the 3 dimensional vibration of space. When it formed, it can send out small waves in all directions regardless. It is more intense in movement direction. If it did not act as this, a laser which is held with an angle relative to eye would never be seen. It seems that eye is extreme sensitive. Also all of the particles in the universe act like photon; because particles are formed by photon and even if you can't see 1 single particle, it vibrates the space in all direction like single free photon in the other name light. This can explain famous the double slit experiment [4]. Even 1 particle has radiation and a color if the radiation amount is enough, because of its natural vibration which occurs because of the heat that is together with energy even if you can't see it. Also particles which move in an order back to back may cause color at the required frequency according to a fixed reference.

Let's assume that $x$ is the distance which the gravitational $f$ force can pull back an accelerated mass and lower the speed of the mass in a $t$ time. For $m^{2} G x / x^{2}=m v^{2}$, it will be $v=$ $\sqrt{m G / x}$. For $x=v t, c$ is the speed of light and $t$ is the smallest time allows this work then it will be $c=\sqrt{m G / c t}$. Here if $c, t$ and thus $m$ is constant as required by uncertainty, then also $G$ must be constant. The total mass of the universe must be

$$
m=t c^{3} / G(K g)
$$

for this small escape time of $t$. The frequency of this mass must be $1 / t$ for this time. Formation is not only along 1 second for the beginning of time. After 1 second, the photon mass must form a black hole for 1 second according to its own rule that photon mass occurs for 1 second. It is a necessity due to mass and energy conservation. All the energy which the universe possess must exist for 1 Planck time in the beginning of time potentially. All the formed energy during this 1 second could be stacked into this small time. In addition works of God is possible when compactness and expansion states exist together. It is possible to create living creatures by using two of both regions. If the waves intertwine and form a particle, it is not possible to create particles like ours in the other region; thus we can be formed by compactness; in this case for $m_{p h}=$ $4 \pi / c^{6}(K g s)$ and $m_{p h}=t^{2} c^{3} / G$, the Planck time must be (28).

$$
t=\sqrt{4 \pi G / c^{9}}(s)
$$

The Planck length for this time must be

$$
L=\sqrt{4 \pi G / c^{7}}(m)
$$


and the constants emerging with the required setting can be found. The mass stated the above as (27) is the mass which does not let light off in 1 Planck time. This mass then has $m=c^{3} / G(K g s)$ mass magnitude as it completes its motion for 1 second for the same radius. The force caused by the centrifugal acceleration emerging due to this mass must be (30) as the below

$$
f=\sqrt{\pi c^{17} / G^{3}}(K g s)
$$

being $r=L / 2 \pi(m)$ for 1 second.

He withholds the sky from falling onto the earth except by his leave.

\section{Quran / Al Hajj - 65}

and

God who raised sky without any pillars that you can see, is firmly established on the throne.

\section{Quran / Ar Ra'd - 2}

verses talk about it. A strong vacuum area emerges due to this force. Universe uses its own heat to work; thus also it loses heat during this work as well; thus space is always cold. The acceleration emerging for this force must be

$$
a=\sqrt{c^{11} / 4 \pi G}\left(\mathrm{~m} / \mathrm{s}^{2}\right)
$$

for $a=c^{2} / r$, and the path proceeded for the reference time of 1 second based on $x=a t^{2}$ must be (32) as the below

$$
x=\sqrt{c^{11} / 4 \pi G}(m)
$$

and is the radius of the universe. As the light velocity made the universe stable for the higher values which are stable, it performs only the act of emergence, and the universe does not expand after the expansion for 1 second. As all actions can be performed as much as total energy of universe or used matter; based on the action performed in the direction of centrifugal acceleration, the mass emerging as a result of this action performed must be

$$
m=c^{12} / 2 G^{2}(K g s)
$$

It can be said that rather nonsense physical phenomena in the universe are possible for such a big mass and they can occur at any place each second according to the total number of the particles emerging as well. The density of the universe must be

$$
d=\sqrt{9 \pi / G c^{9}}\left(K g s / m^{3}\right)
$$

; but the universe can only possess the density when all of the particles of the universe evaporated. The universe has a center and the universe which seems as expanding is caused by the photons which create particles, and also by evaporation as the universe emerges due to centrifugal force. During the expansion, a potential difference can emerge along the space which are along the direction of centrifugal acceleration because of the initial movement, and masses move by photon momentum because of photon friction. Matters' own creation motion which is momentum in the other name causes outer space movement by friction. Also this is gravity. The time differences which are caused by the initial movement in the centrifugal force way and the circular motion of the universe, may cause helical motion. Even if photon wants to move straight manner, time differences do not allow it to move that manner. It may be caused by the initial movement, and even if the universe turns in superposition condition, it always shifts slowly that it should be at the speed of light as some slices as waves and is a second, side effect. Spooky action at a distance of Einstein [5] may also be caused by this superposition of the universe as every action emerges in a time interval as a result of entanglement, single universe mass. As it does not differ for universe that is it a particle, the universe can touch all of them at the same time from the furthest existent distance. For these information, it can be said that

As universe has been emerging due to centrifugal force, finally it must be static actually as required by the conservation of energy.

\section{Proposition 21}

A density difference occurs because of one by one emergence and having frequency of it, and matter does a motion as translational motion. A space contraction can be seen at some points of space because of the mass scatter. These points are denser. A matter which has a smaller density than universe cannot exist. When it existed then it is suddenly excluded and be transformed into a photon or loses its vibration and gains an energy under the Planck constant as uncertainty warned. Because of this reason, some particles may be lost as emerged at the moment they emerged. If we look at the information, it can be said, that the waves intertwined together and they formed the subatomic particles, and then hydrogen by an ignored time difference; thus diffuse of particles is not so hard in the universe in 1 second. All of the work is together and is in 1 second. If many masses cross on the same point at the same time, then space must be collapsed and then it must be swollen; thus new types of elements may be done by photonphoton collisions, and they do not have to be like in our scientific knowledge. Supercomputers will be saver to do it.

\begin{abstract}
After that, he headed to the sky which has been as smoke, and said to it and to the earth that willingly or unwillingly follow my order. Both of them said that we came willingly.
\end{abstract}

\section{Quran / Fussilat - 11}

verse talks about them, and their wills are physical laws. Also the brain should be the transporter of mind as single, and it can be said that as it dependent of the same laws, the mind has a will. It is the same but better than matter as working type changes the result.

As matter emerges by space which is the basic matter, if matter draws extra space during its emergence with different magnitudes along the emergence as a side effect that is unavoidable as its own emergence because of uncertainty like pasty substances, it can move faster than light even though the light velocity is fixed; because matter does not have any other motion except its emergence motion, and it can go faster than light according to the extra gained space. The condition can be expressed as

It is possible to move faster than light in outer space to extent of the extra mass drawn from space during the emergence even though the light velocity is always stable.

\section{Proposition 22}

and it is applicable for the masses covering a range of very minor and very major masses, and is an effect of matter on itself. The universe was able to expand faster than light for 1 second at the beginning of time by this way. If the condition of moving faster than light is calculated and the particle cannot move faster than light for the force which it is pushed by, then this means the particle exists with some subatomic particles together; because area of the lowest energy determines efficiency of a system as stated the above. If a bigger mass 
is in question it can change the condition of subatomic particles with a violence due to gravity; this way mass is drawn from the space with a frequency and the event is like creation. The second effect which is assumed frequency of violence behaves as particle. If energy exists, it is not prevented. Extra space drawn consumes life of matter; because matter leaves a bit of its mass in both of outer space and creation motion behind as required by uncertainty, and at this time gravity is still active as well; thus during circular motion, the photon which forms matter collides with itself in great number of collisions for each second. Matter can only use its own energy for all works; thus the collisions cause evaporation.

Matter has no basic absolute mass particle, photon is included as well; but when relativity warned, photon must be assumed as mass particle as the reference. If this is accepted, Gordon Kane from the University of Michigan needs to pay 200 dollars [6] to former Lucasian Professor Stephen W. Hawking if Mr. Kanes does not kick up a row suddenly. If he can give me the money, please I would treasure it as a souvenir; of course, if I do not sell it for a lot of money via internet. Photon performs the motion linearly that has $L$ wavelength for its helix which has $r=L / 2 \pi$ helical radius; so it does not experience any delay for the Planck length and the Planck time, and maintains $L=c t$ status. The decrease of helical wave radius lowers the linear wavelength; thus mass per volume in an unit can be increased; thus also it is able to increase energy of photon. High energy photons as gamma rays can go through matter without getting interacted with matter in this manner. Energy of a photon which has 1 Hertz frequency must be

$$
e=h v(J s)
$$

as Max Planck derived it [7]. Energy makes a single photon at a smaller time frame, and does the helical motion with a smaller string.

Photon performs a single motion to form particle, and frequency of a photon on a particle surface determines the radius of particle; thus $v$ being the radius of a free particle which does not consists of other subatomic particles, $v$ being the photon's frequency of revolution around the particle, $m_{p}$ being the mass of the particle, $m_{p h}$ being the mass of the photon during 1 second, for $m_{p h} v=m_{p}$ and $c / 2 \pi r=m_{p} / m_{p h}$ free single particle radius must be

$$
r_{p}=h / 2 \pi m_{p} c(m)
$$

This radius can be vary based on the place it exists in space. With another formulation, $h$ being the Planck constant, as it will be $m_{p} c^{2}=h v$, it must be

$$
m_{p}=h v / c^{2}(K g s)
$$

These are hypothetical and indirect calculations for the available values as there is no alternative as well. The sensed force of a particle as the result of its displacement due to a mass must be

$$
f=m_{p h} c^{2} / r_{p}(K g s)
$$

The relation between mass and radius of particles based on action performed must be

$$
r_{p}=\sqrt{h / m_{p}}(m)
$$

; thus particles must have bigger radius; however the gravitation on themselves and re-vacuuming of the space which is already a vacuum in a small time interval prevent it. Considering this it can be said that the free particles which do not consists of sub-atomic particles with small radius and big mass, emerged earlier at the beginning of time; because the same photon stays at a small radius due to the stronger in- ner vacuum, and this can occur when the universe was denser. This affects the destiny of magnetic dipoles. Also particles of the universe may only be formed by the first creation. There is nowhere to escape, and the waves dig the space; thus the photon which creates the particles behaves on the surface as membrane with pressure difference between the inside and the outside of the particles. The vacuum force wants to pull the membrane; but the photon's centrifugal force forbids this. Maybe open helical motion causes and photon does not move on surface helical; thus particles are like beam of a single atom, that the beam travels circular according to its total beam energy and create centrifugal force somehow by the first creation. Maybe it behaves like a memory metal. When the photon mass is assumed at number of the frequency around a particle for all time and do not move based on the mass of the particle, the gravitational effect of the particle on itself must be

$$
f=m_{p}^{2} G / 16 r^{2}(K g s)
$$

These information blink to the Thomson atom model as particles with smaller mass, have bigger volumes. Maybe proton draws electron into the vacuum area in itself and electron forms atom by inflating proton due to its volume; this way orbitals become characterized with specific and certain values with certain coefficients for the incoming electron mass, and when the electron exists the energy of the orbital difference is discharged as radiation. However the fact that Thomson did not mention about uncharged particles can remain outside of usual process of science; because nothing is perfect due to uncertainty requirement but it should constitute with a potential difference and absolutely be polar.

When a particle emerged, space is stretched and magnetic field lines emerge at the light speed to the end of the universe. Ellipticity of the lines changes according to the magnitude of frequency. The gaps between the lines emerge due to uncertainty. These lines and the gaps in-between have different densities and cause heat even for a single free particle which does not consist of sub-atomic particles and not entangled with another mass, or also for a single free photon which rubs space and itself; thus an object which moves in space without acceleration cannot protect its speed forever. When this particle emerged, a magnetic dipole moment exists there. Magnetic features of each particle are different. On surface somewhere always more mass density exists because of the initial movement. Catching up with itself in the other name superposition affects the mass density on the surface; thus the particles which are uncharged and do not have angular momentum do not exist. Each particle has the same magnetic pole and thus the same type of charge; but mass difference can be seen as magnetic poles. Extra gravitation and at last impulse can be seen relative to frequency and thus mass difference. When the spins of particles turn to the same direction and they distend the space in a direction, the same impact is felt much stronger. As each mass has different density when it is free and when it is in a system due to entanglement and uncertainty, magnetic feature changes based on the place where it exists. Electrical charge can be nothing but mass; because matter must be one type due to infinite frequency and deceleration from absolute energy; thus attraction emerges only due to the masses moving in stretched space. This action is performed by the photon-induced static momentum of each mass. Photon rubs the stretched different density space sweetly because of photon's own momentum and the particle which is created by the photon is seen as be drawn in the tension direction; so it can be maintained as

Gravitation is the will to move on stretched space, the photon-induced friction. It is caused 
by photon's own momentum. Nothing attracts masses.

\section{Proposition 23}

None of energy transformations can be independent of the equation stated the above as (18); thus the upper limit of mass focus is all mass of the universe during this gravitational attraction. To be freed of gravitational field or escaping it is not possible. Attraction is reciprocal and as it does not have a threshold value, it affects from the other side of universe or has an effect on the other side of universe at the light speed. For example if escape velocity is taken, for $f x-\int_{0}^{x} \frac{m_{c} m_{g} G}{(r+x)^{2}} d_{x}=m_{c} v^{2}$ the escape velocity in vertical must be

$$
v=\sqrt{f x / m_{1}-\left(m_{2} G x / r(x+r)\right)}
$$

and the result is zero when both of the sides are squared for $f x=m_{c} v^{2}$. The reason is conservation of entanglement and energy. When the action is divided constantly forever by the parts which are equal to each other, the action performed by gravitation closes to all of the actions performed against gravitation, and this is an evidence for single factor of creation which is gravity. Escape velocity for the land vehicles moving on surface can be calculated with the required setting, $f$ being gravitational force, based on $m v^{2} / r=f$. A constant state of performing action is required to conserve the velocity; otherwise, the accelerated mass first slows down and comes back even if it is from the existent furthermost distant; thus it can be maintained that

To be freed of gravitational field or escaping it is not possible.

\section{Proposition 24}

and the situation changes only when entered into the another gravitational field which will balance or defeat this situation and an action is performed against the first field temporarily; thus a fixed orbital is not possible. Even if masses, attraction and thus motion are reciprocal, objects get closer to heavier masses around which they rotate, first move away and after get closer or move away because of outer gravitational effects.

A gravitational effect is also caused by displacement which is circular displacement. This effect causes deceleration of a mass with a period on an orbital. There is a slower rotation due to the concentration of masses on the centers of galaxies; and the orbital heavenly bodies which are placed far to the center rotate relatively faster because of inner mass density which can be assumed as a single mass and circular displacement. Due to this displacement, infinite and zero mass points for $\tan (\alpha / 2)$ emerges over a circle. Where the mass has an infinite magnitude at one point, the point is the point of zero mass or the mass gets its usual mass magnitude which is detected at the end of 1 second; thus a person who throws hammer with circular motion, firstly stores the energy and then secondly uses this energy. This situation is in this way in all interactions. Also a mass gets different densities when it is free or is entangled. This mass is not independent of the system and the system intends to behave as one-piece.

The second effect of this displacement can cause helical motion. To be one of them before or after of two the same opposite works which are done by the same energy like $+F t$ and $-F t$ change the displacement and it can be used for astronauts to teach them space swimming. It is possible to reach jet fighter speeds and more. Otherwise, swimming in a liquid would not be possible as well. Already, a marble which starts to turn around a center string-dependent manner will pass from a higher place in the next round, and also it constantly climbs vertical manner between these two rounds as well; because the work done by the marble against the gravity increases. The same event can be seen in the universe. The center can be assumed as a sun; thus for the reason the orbital stuffs are assumed as the marble. I did not calculate; but if it is possible, it will be the effect which is the effect on the system on itself; because as stated the above, nothing is perfect and to be before or after of one of the works is important.

Radius of particles and thus their masses are vary due to intensity of the interaction during gravitation. A real decrease or increase emerges for masses; however this occurs according to the relativistic energy transformation formula (18); because even if gravitation is acceleration, it is as a motion with constant speed for 1 second when masses do not accelerate or have not been accelerated, and it is always $a=v$ forever actually for each small period of time in 1 second as well for $t=1$ second based on $x=v t$ as average. Some minor changes in the volume can be observed; however, this occurs as length contraction. $m_{0}$ being the standard mass at the infinite distance from a gravitational field; $m_{p}$ being the mass in a planet; $a_{p}$ being the gravitational acceleration on the planet and $a_{s}$ being the gravity acceleration in space; for $m_{p}=m_{0} /\left(1-\left(a_{p}^{2} / c^{2}\right)\right)$, the radius causes the ellipsoid at a certain height from the ground or in space must be

$$
r_{s}=\frac{h\left(c^{2}-a_{s}^{2}\right)}{2 \pi m_{p} c\left(c^{2}-a_{p}^{2}\right)}(m)
$$

It starts drawing an ellipsoid. Even if time is relative, it can not be detected as varied around, near or surface of planets; because time must be accepted together with space; thus if time shrinks, then energy must shrink. Variable is total energy and thus is volume and mass. Every existing physical feature absolutely changes all together with these changes at the same rate. For example, the conductors of an atomic clock would be more insulating at the closer place to the surface of a planet; because electron mass is inversely proportional with electrical conductivity; thus an energy producer produces less energy, and the clock stays behind because of the reasons. By the same reason, astronauts need more food; because they need more molecular reaction energy. When they run in space, the same energy will occur after more molecule use to protect the old situation. They should eat appetizing foods and for example for Mars, we must let marriage. By the same reason, ionic bonds will be weak in the space. Centrifugal force is enough to protect bones and also is good for feed except metabolic pressure; because it has the same effect on the particles like gravity since gravity is constant speed motion for 1 second even if it is an acceleration.

Particles move back and forth in a rather minor length due to momentum of photon in rather dense area during gravitation. This point is now the area where mass impulse is rather dense. Rotation of the spins at this moment should not be neglected. Moreover, also during the moments when the matter is not there, the status of inertia of the particles is destroyed together with the space which has been fixed and healed, and a mass impulse effect can be sensed.

Each existent action is absolutely at the light velocity. As required by uncertainty; photon must leave some part of its mass where it has passed during the motion which it does with a frequency, and the same is valid for all spatial motions of all masses. At that time gravitation is effective on itself as well. Due to photon's status of superposition with itself on particle, photon must lose its energy and must spin the particle somewhat according to non-flexible friction or if the friction has a high frequency then according to a collision, and causes a centrifugal force against the radius of heavenly bodies especially the compact ones, and it must be included to the calculations. 
This effect becomes stronger together with the increasing frequencies because of the decreased radius for constant velocity. As the effect affects the life of particle, also it must stop or reduce the vibration of photon utterly at one point as required by conservation of energy, and at last the mass must evaporate at an amount all of the mass is included due to the intensity of the interaction; however the total energy of the universe does not change. Whether one effect occurs prior to the other or does not changes actions. For example, as it was said when a $f t$ action is performed in space, if the same $f t$ action is performed at the reverse direction at the end of $t$ time, the displacement is different than zero. Also this is good example for famous self-running magnet motors. The same is acceptable for back emf with right coils. If you want to take an object from A to B in space, this work is done by first acceleration and secondly slowing. Otherwise, the object accelerates forever; thus the first work is acceleration always. If you want to shorten the work in the point of time and thus distance forever, nothing changes and the rule is still valid. Similarly, in an electronic assembly, the current is the first for any time if you do not use the matter I mean even there is no electron flow between atoms; thus back emf is the second work, and in the right electronic assembly, the second work's effect can be passed maybe some high frequencies because of smaller distance requirement. I think it can be done by many coils by using the coils by turns for 1 second. Also, we can vibrate the electrons without the flow and thus resistance with resonance. The sum of the vibration occurs as magnetic pulses and the current can be created. Generating or transmitting electricity without resonance is always our enemy. If we turn back to the subject, there is nothing but the event. Even if this condition causes the magnetic dipole for the photon moves on the surface, the effect of mass left behind by the photon changes the magnetic dipoles by the same reason of evaporation, and the same particle can behave differently while in a different mass, temperature and thus in a different frequency magnitudes emerging.

When the motion of photon in a circular motion reached 1 photon mass for the mass which it has just left behind, it can be accepted as flexible collision as its mass can emerge during the time of 1 second for constant single way speed, and it evaporates at the amount of extra action due to its uniform energy. 2 photons whose frequencies are 1 in space are assumed as they perform flexible collision. 2 photon masses are detected at the point of collision during 1 second, and both of the photons evaporate at the end of this time. Due to the rotation in volume for $2 \pi r=L, L / \pi$ being the width of photons and $r_{p}$ being the radius of the free particle, there are

$$
2 \pi^{2} r_{p} / L
$$

transition places. The photon with $c / 2 \pi r_{p}$ frequency for the radius of the particle forms the $m_{p}$ particle mass at the end of 1 second. $c / 2 \pi r_{p}$ frequency and $2 \pi^{2} r_{p} / L$ frequency must be the same for the maximum entropy as relativity warned as there is no disorder; however, $c / 2 \pi r_{p}$ being the frequency of this particle, it is

$$
r=\sqrt{L c / 4 \pi^{3}}(m)
$$

emerging for $c / 2 \pi r_{p}=2 \pi^{2} r_{p} / L$ for a single radius. To achieve the same condition with changing radius, the photon mass and the light velocity must change either separately or together with a proportion; thus $c / 2 \pi r_{p}$ being the frequency of the particle; for $\left(c / 2 \pi r_{p}\right) /\left(2 \pi^{2} r_{p} / L\right)$,

$$
v=c L / 4 \pi^{2} r_{p}^{2}(\text { Hertz) }
$$

must be the frequency of the photon whose frequency is 1 . If it is not, it means there is a superposition and so a flexible collision with photon itself.

$$
m=m_{p h} c L / 4 \pi^{2} r_{p}^{2}(K g s)
$$

is the amount of the mass evaporating at the end of 1 second if you assume that particles do not lose energy during the collisions, and if you assume that it happens suddenly as well; so they lose at the end of 1 second, that it does not happen by this way if evaporation does not happen as an energy storing. By the way, the mass thus the radius change at the end of this period. If edited for $r_{p}=h / 2 \pi m_{p} c$, then it must be

$$
m=m_{p}^{2} c L / h(K g s)
$$

The energy evaporating at the end of 1 second must be

$$
e=m_{p}^{2} c^{3} L / h(J s)
$$

The calculations for the total life must be done again and again for each second. If the energy evaporation on the focal points and thus the loss of mass occur with massive periods, this loss can be assumed as stopped at status of the highest assumed entropy even though the annihilation actually continues on these focal points of the universe as required by uncertainty. The mass which will lose its total mass at the end of 1 second must be

$$
m=h / L c(K g s)
$$

The radius of this particle based on $r=h / 2 \pi m c$, must be

$$
r=L / 2 \pi(m)
$$

and verifies the above mentioned findings. The energy of any particle in any mass which does not consist of sub-particles and is not entangled with a system must be

$$
e=h / t(J s)
$$

As matter can perform action as much as its total energy, it must use its own energy even during an extra mass sensed in a time interval in superposition with itself without any external effect, and its energy must decrease as much as the energy which is spent by the formation photon of matter at the end of this period. Matter can only use its total energy even if it does work or made it worked; because for both of the conditions, matter must be has been working. Instead of flexible collision with constant speed, the gravitational effect on itself can also be used in the worst status to prove this as a perfect evidence. A small string emerging in each Planck length imposes a gravitational force right before the superposition. This force will be

$$
f=m_{p h}^{2} t^{2} G / L^{2}(K g s)
$$

As collision occurs at each Planck time, it must be multiplied with $1 / t$ and as $1 / t$ number emerge in each string during 1 second, it must be multiplied with $1 / t^{2}$; thus

$$
f=m_{p h}^{2} G / L^{2}(K g s)
$$

force is the gravitational force emerging during 1 second for the photon reciprocally colliding or moving around $L / 2 \pi(\mathrm{m})$ radius. It is completely equal to the force which creates the photon during 1 second for (29) and (25) values which were calculated the above, and it performs the same action in the reverse direction and consumes the energy of photon. This decrease can be as energy storing, and actually should occur as instant weakening under the threshold value due to the decrease in small strings' masses which emerge in each Planck time as it does not actually have a threshold value. Meanwhile, all the performed work converges to the gravitational work and becomes equal due to impossibility of escaping or 
getting rid from the gravitational statement has been observed the above; therefore it will not be wrong to say, that the only truth is gravitational force. Heisenberg's uncertainty mathematical interpretation, which is

$$
\Delta x \cdot \Delta p \geq \frac{h}{2 \pi}
$$

equation [1] is not for $h / 2 \pi$ but verifies it for $h$ with a right interpretation. $h$ is the Planck constant and the smallest distance which a matter covers should be Planck length $L$. The smallest distance which can be taken is the circumference of a circle due to the circular formation, and the radius of the circle is $L / 2 \pi$ which is the photon's wave length which has $h$ energy; for this reason, $h$ cannot be divided by $2 \pi$ under any circumstances. For formed mass over 1 second, acceleration will be $x=a$ based on $x=a t^{2}$. As $h$ becomes Planck constant with $(J s)$ unit, being $c$ is the speed of light, $h=m c^{2}$ and $\max \geq h$, uncertainty equation is going to be $a x \geq c^{2}$ which is timeless velocity equation for the speed of light that is entire universe's constant formation speed. It will be $a=c$ for $v=a t$ along 1 second; the distance taken for 1 second will be $x=c$. Here acceleration can be counted gravitational acceleration $g$ as relativity warned about 1 second of gravity that is equal to constant velocity motion for $a=g=c$ as it is actually still equal in the smaller periods of time by the nature of motions as well; thus constant velocity which is the assumed single reality of universe and the resulting force are equal to themselves. Maybe it can be said, that it was assumed and calculated over an assumption; but it can be verified. Firstly in order to perform work, matter must stand and stay together even if time and thus mass decrease for smaller periods of time, in every moment of matter's shrinking period due to uncertainty requirement, even for matter's assumed the smallest mass part. The work done to keep matter together will be equal to kinetic energy; because work done is equal to kinetic energy, and further is equal to total energy of matter; thus matter cannot have more or less mass or energy than this work. When mass owes its existence to only one work, then it has to owe its total energy to the same work as well. Matter is certain as much as its existence, and total energy determines this. A free particle can be uncertain until the photon that has 1 Hertz frequency, $h(J s)$ energy and forms particle; thus the mathematical presentation of uncertainty (54) is very appropriate, and to be the only truth of gravity is very sensible because of potential differences of God's creation and forcing which are always against gravity that means recovery wish of space, cause gravity and are equal to gravity. If it is accepted, then many calculations will be child toy especially for evaporation. It can be done directly over gravity.

Furthermore, if it is necessary to make a mathematical construct, $g$ is the gravitational acceleration that it is assumed that $a=g=c$ for 1 second. For the constant speed movement requirement, it will be

$$
g=c=m G / x^{2}
$$

If $G$ is taken for the smallest distance $x$ which allows this work to be happen in the distance, then it will be $G=c x^{2} / m$. Hereby the distance is the Planck length which is the smallest distance allows the work to happen, and if $G=c x^{2} / \mathrm{m}$ equation is arranged for $x=L, m$ will be the photon mass at $4 \pi / c^{6}(K g s)$ value, it will be

$$
G=L^{2} c^{7} / 4 \pi
$$

If $G$ is taken from the above calculated (29) with Planck length, it will be $G=L^{2} c^{7} / 4 \pi$. The result is equal to (56); thus the speed of light $c$ is an absolute gravitational accel- eration, and is the source of all energy. As a result of this information, it will be accurate to say that $\Delta x \cdot \Delta p$ equation is the gravitational potential energy as on (16) over uncertainty equation (54), and matter does not have any other energy to do another work.

$m_{u}=c^{12} / 2 G^{2}$ being the total mass of the universe, the frequency of the universe must be

$$
v_{u}=c^{12} / 2 m_{p h} G^{2}(\text { Hertz) }
$$

As the difference in time is rather minor, it has been neglected in the calculations stated the above. The linear and from surface to surface reciprocal gravitation effects on itself have also been neglected. As gravity-induced decrease in helical circulation radius changes mass and wavelength at the same rate, going to disorder of any mass occurs at the same rate for entropy. This is the effect on itself of matter. Moreover, if it is not a smooth sphere and if is a moving object, calculations are done over the surface area of the ellipsoid emerging. Somehow the age of the universe can be calculated by observing energy changes of the particles with the calculations stated the above very definitely by using single, free particles which are not composed of another sub-particles or are not in a system, like electrons. Maybe also by periods and energy of the gravitational waves; because the waves spend their energies when they passed through themselves; but we need superior computers to calculate it to help us I think.

For Schwarzschild radius based on action performed by the kinetic energy correction, $m=L c^{2} / 2 \pi G$ mass is the mass which emerges with the smallest radius $L / 2 \pi$ for $2 \pi r=L$, and is based on $f x=\left(m_{1} m_{2} G /(L / 2 \pi)^{2}\right)(L / 2 \pi)=m_{1} c^{2}$. The radius emerging for increasing masses increase at the same proportion with the mass; thus all of the black holes which are cosmic or formed with single free particles must stop their vibration at the end of 1 second, and then evaporate. The heavenly bodies called as cosmic black holes are the pieces of stars, which gets smaller due to fierce explosion and the compact objects which cannot be freed of their own gravitation, and stop vibration of the light at the latest within 1 second based on their severity. Also another black mass which the light cannot escape and is not Schwarzschild black hole must be the mass which gravitational acceleration will reach objects excluding photon to the light velocity along 1 second according to the relativistic energy transformation; thus this heavenly body considering $a=g=m G / r^{2}=c$, has radius of

$$
r=\sqrt{m G / c}(m)
$$

It becomes visible together with evaporation by slowly opening and there will never be a sudden volume increase. An increase like this only can occur in Schwarzschild radius black holes. If the mass increases then a dim occurs in heavenly bodies, stars are included; also compact heavenly bodies are denser, heavier than itself for the same particle number if you open it because of the particle radius change; so gravitational force magnitudes change for the same number of particles for a fixed distance during expanding or becoming small. Matter is deemed to be performing an action if it performs an action or is being made performing an action; and matter can be used only as much as its existence; thus all masses including photon lose their energies with gravitation, and the objects which are at a farther distance from surface of a planet have longer life than the surface objects. The mass which does not let the light off with this radius (58) can be assumed as it does flexible collision each second; this way the photon at a frequency at the end of 1 second continues its path as vibrations under its threshold value in space by losing its energy forever. As it spends the energy of light, major differences in heat emerge 
from the center to the surface in such objects. Considering this information it can be maintained as

The compact objects which can be jammed down to the Schwarzschild radius can form a black hole that evaporates within 1 second. The masses which live longer than the period of 1 second are not Schwarzschild black holes, and do not let the light off. They are possible and have much bigger radius.

\section{Proposition 25}

A Schwarzschild black hole may not has been emerged yet in the universe due to the evaporation and Coulomb push force which would emerge because of the smallest radius required for forming a black hole centrifugal force-induced push is included as well, and maybe they are the natural results of each other. Moreover, the central pressure and the mass do not increase at the same rate. The pressure decreasingly increases; because there is always an opposite gravitational effect; so also the heat does not be enough to heat the star, and the wavelength of the star does not increase by the increasing mass at the same rate. For example, a part on the surface applies an opposite gravitation to another near center part with gravitation at the displacement way. In order to understand this intellectually, a surface part of a star can be converted to a black hole, and expose that black hole attracts the star from a close distance. When this happens, the entire mass of the star which is in the black hole's gravity withdrawn at the same time but it begins to lose from the surface. The only difference between the conjoined object and fixed objects with a distance is the magnitude of the interaction. The mass in thought experiment is a black hole; but an ordinary part of mass only changes the size of the interaction. It is calculable by some marbles which have the same mass magnitude and size, and are aligned linearly, by looking at the pressure which is on the central marble. It may be assumed as an evidence for fusion reaction possibility which changes with pressure, heat-induced changing magnetic dipoles, distance, spinning around oneself by particles' own photon-induced self-collisions and length contraction. This is the same thing with cold fusion, and the difference is the increased possibility by the stated factors. In the light of this information, it could be stated as

There is always an opposite gravitational force along with gravitational force.

\section{Proposition 26}

Matter has a blank structure even for its infinite little particles as required by uncertainty; thus each effect can only affect from a range. Whether the range is the existent biggest or smallest length, it can only change the time of interaction and its intensity; thus matter is constantly in a status of reciprocal friction at the light speed with the objects in the universe. During these frictions, the gravitational force on an object in the field of attraction is not constant due to its blank structure and the intensity of the distension it creates in the space has changing potentials. This cause a gravitational torque which has a certain magnitude. Meanwhile an orbital motion must emerge at the same way of the force which creates the torque; then

The masses have orbital motion effect on each other in the same direction with the motion of the force which forms torque together with the gravitational force.

\section{Proposition 27}

can be said. The mass density in center of galaxies can cause a different spiral structure by applying this torque while spinning around itself. The fingers may be like bended vane blades in this spiral. It does not have to emerge with center at the same velocity. Also each effect is at the light velocity; thus these fingers are affected at different times. As it can be understood by the information above, photon is the transporter of gravity as photon is an effect transporter and as is the transmission, transmitting speed of free space as a photon is a potential difference in space, and it can be experience a refraction by magnetic fields and gravity-induced different density space as light is refracted by different densities and can go circular by some deflection periods. As two space objects can do a free fall around themselves by circular, helical motion, also Galaxies can be had been formed according to center mass's rotation way. This is the same with ellipticity of planets and stars which is caused by axial centrifugal force; thus if a perfect sphere hydrogen smoke forms a center mass, and if the mass starts to turn in one direction axially which is especially caused by particles' own momentum-induced friction, if you assume that there is no other object in space, then torque causes a centrifugal force for the outer space objects. The objects which are the rest of the sphere and are perpendicular for the turning way are directly attracted, and the sphere turns into a 2D heavenly body like galaxies of course if you assume they are created by a nebula. The same may be acceptable for the total of the universe, and it can be said that the universe may be flat as well.

The matter emerging at the light velocity performs each action at the light velocity as it responds to each effect at the light velocity as well. For example, when a fire emerged, the increasing vibration together with the temperature can affect another particle by friction of the particle's frequency in the gamma frequency; thus as it can be variable, matter can perform black body radiation at visible light frequencies even for the smallest period of time and turn into lower frequencies quickly at the same velocity according to the particle's place of existence and to its type. Also by this reason, pressure causes heat for any matter, and if you can enter the resonance with a particle which is radiating at the same frequency at the reverse direction by magnetic field, you can cool it according to changing vibrations in time as well. We do not need to heat materials if we have enough pressure and can use the heat for constant energy resource. Some strong bonds and crystal structures can cause abnormal heat at small amounts as well. Black body radiation must be the natural vibration of matter and forms atom as a fire ball because of the surface photon in the other name wave, and is induced by its emergence motion which changes with temperature; because matter always together with the heat which is included to total energy; thus also matter cannot reach absolute zero; because velocity and so energy become zero. Transparency may be depended on the natural vibration as well together with electrons and atomic structure, and external energy depended color which is caused by the sun or house lights etc., internal energy color which is caused by external heat and named as black body radiation and lasers are completely different things even if there is an intersection. During the black body radiation, since matter can only use the energy which radiates from its total energy, $e$ being the radiation energy throughout the radiation, its frequency being $v$ and for $e / c^{2}$, matter experiences

$$
m=h v / c^{2}(K g s)
$$

of decrease in mass; thus increase in its radius and volume. Considering this information, it can be maintained as

Black body radiation is natural vibration. It causes decrease in mass and energy at the same 
proportion with the radiation energy throughout the radiation.

\section{Proposition 28}

At that time the matter can be polarized, and an electron flow by the other expression an electron jump which is the same with lightning least between two atoms must emerge in a correct electronic assembly. Also black body radiation can be a factor for photoelectric effect. If the extra radiation from outside be focused on a particle as a laser beam like in laser fusion systems, since matter can perform action to the extent of its total energy and since vibrations will be deemed motion, it turns the matter into photon by vibrating in the emergence frequency according to the relativistic energy transfer. Length contraction occurs based on the mean motion during that time. Vibrations are accepted as relative motion; thus the transformation is done by the relative transformation of energy equation which stated the above as (18).

Magnetic dipoles always shifts since matter stores energy and there is no absolute threshold value and thus because of evaporation as required by uncertainty. The values which are smaller than the assumed threshold value are collected and affect particle dipoles. Particles are detected like they spin around themselves slowly as an illusion. Maybe antimatter is formed by change of creation timing. For example, a big shock can do it. The shocked particle experiences a big radius increase in smallish time interval and then the particle allows the light which is produced by the collision to escape. As the light escapes, then the particle get a smaller mass. As the particle get its old mass when the radiation stops, then it is formed with different formation time. Maybe the particle gets itself to right destination according to the condition of the space. Also maybe the time difference causes an explosion if two opposite timed matters collide in the universe at a point along 1 second when they are inside of each other even if they are not similar in shape, like opening a DNA spiral; by this way, half of the universe is antimatter which is equal to matter amount. Matter is antimatter sometimes, and at the same times antimatter which is the other side of the universe is matter, and the condition changes always during 1 second. Maybe not $50 \%$, and maybe more because of the transition moments; but there is always antimatter. Space is neutral for both of them as it collides with them by a friction every second; but its effect can be assumed as zero even if it is not, and I hope the scattering had been in a perfect order. Otherwise, we may detect two galaxies while they are eating themselves in theory if there is a special spin effect although matter dependent of space which emerges with time difference. I think this is a far possibility.

Abrupt differences in heat can cause explosions due to the momentum of photon. Moreover the initial and the final energy values of matter can be different than the mean action; because each action is within a period of time as required by uncertainty, and matter performs an emergence action and goes through change in constantly changing environments and events; so a period of time is required for matter to place itself accurately in space. As these impacts emerge due to matter's own action, the status of matter can be turned into the status of performing action with lower energy, and continuation is possible. We can use matter's own motion of creation by using an incomparably energy and we can force to make it worked for us. For example, in a wound conductor as the energy storing capacity of the magnetic field is higher than the resistance, when the flow which feeds the conductor is removed, a bigger flow can be induced in the reverse direction and a constant absorption is possible by some repeats at some frequencies. The work which is done against gravity helps the pushing and they do not change at the same amount due to distance. If the mass structure does not felt as the result of the formed equilibrium of forces, then forces are gathered by a wound coil, and a growth is provided by $L>R$ status. Volt/d increases for the coil, that the coil can be assumed as a particle as well. Positive and negative EMF can be used as well. Those are gaining from force in the other word voltage, and the system should not only use the energy source. This can even be achieved by using conductive metals by resistance difference of matter. Magnetic flux on a stationary roll is absorbed at a frequency with a material which encircles around the fixed magnet and by using the fixed magnet's energy and changing the magnetic flux on the fixed coil. Electricity can be generated by heat welded absorbing energy by voltage which causes kinetic energy in magnetic plasma environment; existing energy increases by square of the voltage. Apart from these, electric can be produced by vacuum energy by temperature difference by semiconductors or by magnetic pulses which is achieved from potential difference in time in a drain time of current when it behaves like a permanent magnet.

\section{Information Status of Matter}

Information is not absolute. If you assume that God created all the information as one by one with the time differences between infinite information then God must be had been created as one by one; because as stated the above, if a work is in a time interval then an absolute must do the work instead of the worker. As the condition is this, also infinite information cannot be without beginning which is not created as well; because if the information is without beginning then it must be absolute; but as stated the above, there can only be an absolute who is certain and is not uncertain. Here, a third option point exists which is different than physical facts which form our logic. The third option is not the third option which is different than identity and contradiction of logic philosophy. The third option means that infinite information is not without beginning together with and as God, and God doesn't create information one by one as there is no time interval which is God has not known some information yet. Otherwise it is what philosophy of logic says. This means that it is if what it is and if what is what known it means no problem. The third option of philosophy of logic is always impossible. To be true and false at the same time status is impossible; because it holds both true and false states, so it depended on the definition of to be true and false. As true and false, even if they are relative they are opposite to each other at last. There is an entanglement, and the assumed basic mass forms all of matter one by one at an incredible frequency. Superposition which exists because of uncertainty namely matter which is reaching to itself, and the state of dead and live cat of Schrödinger namely preparing of information is not fuzzy logic as well. Information always exists in both state; but as nobody can know an information untimely manner aside God, the work being as resistance for God because of time and the information is prepared and created one by one as far as we can see in Isaiah of Torah, "He who brings out the starry host one by one" is one by one emerging as it seems. More in, "and calls forth each of them by name" is required by conservation of information; because God cannot remain indifferent about his creation. His words are infinite; but maybe he only knows them by numbers. If we say something about the words additionally, he must create us from the beginning; because we are dependent of limited sounds according to alphabets and assumed new letters; thus the sounds. There are limited combinations. As words are limited, it means any theoretical and practical informations are limited even the not related ones are included and it shall finish one day, and we shall be doing the same things again and again. 
The infinite can know space until the smallest detail of space; because information can only be created by the infinite, and information has energy and is spatial. Because of knowing by infinite precision state, all of the infinite information can be located in the infinite small place; thus all of the infinite information doesn't surge and has no energy. Matter is not created from nothingness; but also it is not absolute as well, because as stated above there is no time interval which God has not known yet some information. Matter was created from imaginary time. The condition can be stated as

The matter which is in imaginary time does not surge as it does not have energy.

\section{Proposition 29}

The third option of the article has the same meaning with Hawking imaginary time [8]. By the concept of the imaginary time, we can stay away from the beginning of time; but when this is done, matter is not seen as without beginning; because actually there is nothing but God himself, and we must assume creatures as virtual. God himself is included to his infinite information; thus all of the infinite creatures passes in God's knowledge as the infinite creatures which were created by God. There can only be 1 absolute because of uncertainty. The situation is not as saying "My wealth has been given to me because of a certain knowledge which I have." of Karun as far as we can see in Al-Qasas of Quran. This place is also Muhyiddin ibn Arabi's A'yân-1 Sâbite [9] place.

He is the creator who created the sky and the earth when there was no sample for them. When he has no partner, how can he has a child? Whereas he is the creator of everything and he knows everything.

\section{Quran / Al-An'am - 101}

verse can be assumed as an evidence for the imaginary time.

According to the imaginary time, time is relative and never past, and never will be. Created ones are always at the same place and at the same time; but they are in a state of flow and time gives a reference right this point. The energy that is spent by creatures is $m v^{2}(J S)$ for a creation speed of $v$, and they spend the same amount of energy forever or in the infinite sum, since everything is the element of infinity; thus it can be said that

Time travel is not possible.

\section{Proposition 30}

Let us assume for the moving objects, time passes faster or slower somehow. As gravity is like constant speed movement and like it gives momentum and thus weight to masses, somehow also let us assume that gravitational areas affect the way of time flow in some directions as next or back. In the mix in any condition, as time is the displacement duration which occurs together with energy without doubt, it must affect the energy of matter. If this is the situation, then for back time travel, matter gets zero energy as time thoroughly slowed down and finally became zero; thus matter loses its total energy for the next move. Already as God has to know infinite information untimely manner, if slowing time is possible, disappearing of matter afterwards is not possible as stated the above. Information shrinks forever; but it never turns back. Also it will not be possible as crossing, jumping from zero point is not possible in next time travel move. Also again for these conditions which are moving objects or big gravitational fields, if it is chosen one of the options of time passes faster or slower for back or next time travels, we need a gravity which must decrease and a gravitational field which we must stay away and have infinite distance from it. As faster free space moving objects and the fixed objects which are in strong gravitational fields are the same because of nature of gravity, in any mix, we need increasing gravity; but also at the same time we need decreasing gravitational force. This is a paradox. All of these things sign to a now; thus neither back nor next time travels are not possible. In summary, space and time is not independent of each other. If you want time travel, then space must be lost and then there will be no next even if it is for past or future. The condition can be maintained as

Travelling in time is only a dream.

Proposition 31

and it is scary. As creating past or future time is a paradox for God, it is not possible even for God as well to take an object to the old position if it moves.

Before creating a thing God can only record it as information, due to the potential difference which could occur from the infinite, and can create the thing that God wants to create, even if there is no time actually as required by preservation of information.

No calamity that hits the earth or you but is recorded in a decree before we bring it into existence; that is truly easy for God.

\section{Quran / Al Hadid - 22}

and

Nothing hidden in the sky or on the earth but is recorded in obvious decree.

Quran / An Naml - 75

verses talk about it. There must be two different record places because of people's choices, at the choices God's different wills and already requirement of conservation of information; but finally all of them must be in a sky book that is formed as matter.

In a book preserved.

\section{Quran / Al Buruj - 22}

verse talks about it. As information is not without beginning, creating from nothingness requires this.

Meanwhile information could not be eternal. Create out of nothing first time requires this. Information of God could be seen when asked him in the assumption of everything is known like God; because information could only be created from God himself and it is needed to be eternal to ask questions. Until that time, it seems God does not know, but knows. It seems so, because there is no need to remember or no forgetting issues, due to having timeless and infinite energy. He does not try to sustain the information all the time.

According to category of information, everything is not the same thing; therefore there should be a right and a wrong. In any mixture, the basic polar extinction rule of universe has good and bad or right and wrong division, and there could be no anomalous action. If there is a created one, it could be limited because of uncertainty and subject to a rule, either it is known in an infinite time or not it has a using manual. Goodness and badness are relative as well. If God was unreferenced infinitely bad, the rule of the created ones would has to be in terms of God's morals; because God, the owner of infinite ferman and discipline could not do the other. If God was infinitely good, there would be the same; therefore God is always good; because for example to be not able to lift a big weight would seem as bad thing; but at the point, we can say God can create good or bad because of bad creatures. Bad is in his mind untimely manner; but you created them. Saying 
"God doesn't create the bad" is claims of divinity since nobody has absolute creation power. The things will be talked about or be done could only have a meaning in the infinite mixture with God's saying. The works which will be talked and be done only make sense with God. Because of the using manual and will, testing the owners of mind is an obligation as required by infinite justice; because limited ones are not perfect. Perfectness increases forever but never disappears. If there is another I with limited free will, the owners of the I must be tried; because good can only be gained and bad can only be cleaned by working and learning, and in a time interval which has to be limited because of the right of good people.

Then began satan to whisper suggestions to them, bringing openly before their minds all their shame that was hidden from them, he said that your Lord only forbade you this tree lest you should become angels or such beings as live forever.

\section{Quran / Al A'raf - 20}

verse talks about it, and shows us devil is not an easy enemy. You cannot wait forever. Also he cannot create forever, infinite number of people as any time is now, that there is no absolute flow which creates a paradox in our minds like "Never comes its end because there are infinite flows, times."; thus God can only ask "How many?" as any time is now. There can only be 1 absolute and the infinite cannot be created afterwards, one by one as stated the above. Little and the most are the same at this point.

So that, we wrote to Children of Israel that if who kills a life but response of a murder or breaking order on the earth, they get become as killed all people; and if who cherishes a life, they get become as cherished all people. I swear that although our messengers came to them with obvious evidences, after this still most of them really got become spendthrifts who go to extremes.

Quran / Al Ma'idah - 32

verse talks about it.

And soul is God's imaginary blow from himself. People sometimes could act against their characters and also change their genesis positive or negative manner; but God knows everything as he has infinite ferman and discipline as he knows all the information timeless manner and became only one thing, and doing things against his own morals which knows them informationally, is a paradox. Because of the imaginary time thus could not be uninformed status, as he knows that he cannot be he forces himself an imaginary feeling and thinks creatures; but this is a work and creates time, and only possible with creatures. It is other than God himself and is virtual part of God. If God wants to see a real existence, all the creatures lose and God only detects himself. God knows that what do creatures do and feel; but he does not know how is to be a human being certainly. All of them are together and creatures exist, even if they are not absolute. Creatures seem like they cause permanent damage in God's brain if we assume that God has a brain. This damage is like an absolute damage which could not be healed with physiotherapy even if it is not an absolute damage and is easy to remove. The owners of mind has free will even if they are limited and dependent, and the free will is limited as well. Creation is more than a puppet play; because God's destiny is not being uninformed and knowing the infinite information together with creatures, and the destiny of creatures is being a part of God, even if they could not be infinite and eternal. All the infinite information is known untimely manner by God; thus destiny is an absolute reality even if it doesn't interest creatures as destiny is knowing of God. People must act according to the some known, certain rules. They cannot act according to destiny that they do not know anything about it. Destiny belief is equivalent of God belief. Furthermore, God is similar to people at the point of longing; because every time is now, and he has not been created everything yet, and won't be created all of them one day. People cannot see God completely when they are alive or after death as well. Limited human can see God by parts, and human forgets his old parts. Human cannot combine the parts to understand. In theory, human can see God by naked eye even in the world; because there is no difference between soul and matter or life and death. Just they are some energy thus condition change.

It is not possible to detract anything from infinite or bring in; because everything is the element of the infinite. As all the infinite information can exist by God, God can only create by his own untimely information. During the process, God's names occur. For example, if creature has hunger property then God gets feeder name. As God knows everything untimely manner as stated the above, he has been gotten all the names that can be occur untimely manner. He does not create from nothingness; but he unhides.

He raised its size, and he gave it order and perfection.

Quran / An Nazi'at - 28

verse talks about it. Creatures are like emerged from mold, and creatures can have his all names included the biggest known names like creator as well; it can be said for perfect people if you know the rule, and relatively. God did not create any word for only himself. Existence is transfiguration of God. If humans deny them, they deny themselves and become unbeliever.

Then God said that let us make mankind in our image, in our likeness, so that they may rule over the fish in the sea and the birds in the sky, over the livestock and all the wild animals, and over all the creatures that move along the ground; so God created mankind in his own image, in the image of God he created them; male and female he created them.

Genesis 26-27

verses talk about it; but human can only create by God's creation.

Is it not his to create and to govern?

\section{Quran / Al A'raf - 54}

and

But you cannot will unless God wills.

\section{Quran / At Takwir - 29}

verses talks about it. "I'm God" thinking in unity of existence idea means that I closed to God's morals and have been a limited but clear mirror for him or it means I'm a part of the infite as an expression. Göktanrı in the other name Skygod idea is the same idea and as it is a perfect God belief if you do not count the corruption, it can only be a prophet doctrine. Sky does not mean God; but it means God in the sky who is in his throne.

There never was a people, without a warner having lived among them.

Quran / Fatir - 24 
verse may be an evidence for it. Every object around us is heavenly body.

$\mathrm{He}$ is he who created for you all things that are on the earth, after that as he established the throne on the sky he constructed it as seven skies.

\section{Quran / Al Baqara - 29}

and

He sits enthroned the above the circle of the earth, and its people are like grasshoppers. He stretches out the heavens like a canopy, and spreads them out like a tent to live in.

Isaiah - 40:22

and

God reigns over the nations; God is seated on his holy throne.

\section{Psalm - 47:8}

verses talk about it. As there is no emptiness which is without energy because of entanglement, if all infinity is assumed as sky then Göktürk in the other name Sky Turk or God Turk means Turk who closes to God's morals, melted in God and has been clear mirror for God's names. This is an expression; thus when somebody says sky, then it is not only understood as the sky of the universe. This is as saying "I'm Anatolia" of somebody or saying "I'm Turk" if they are from Turkish land even if they are from other nations at that time. 'I'm going to Greek" has the same meaning as well.

As we cannot wait for mathematical calculations from a refrigerator, in the same manner we cannot wait from God for another which does not exist. The unit which does this expectation seems as mind. It seems that mind is an evidence for itself as it is a witness for all thinking, thinking is included as well as the biggest and holy act. It does distinction between good and bad according to the using manual that occurs because of uncertainty. During the action, it becomes grumpy and falls into arrogance when it is in the hands of God like a cattle animal. Arrogance is a mental disease that we must run away from it. Mind occurs during interactions between God's names and is the name's interaction healthy, and actually it must be the result of some main names. If it does not work healthy otherwise it becomes arrogance. For example when live name is manifested, a rock can feel like a lamb. When some names as scholar and informed are manifested, a rock can talk to you and can save information on its memory and can make warn for you. Mechanics of mind is logic, and logic can develop as it changes. Different types of lives have the same logic structure amongst themselves in different groups. For example, if you give an order like "Let us climb the tree" for a fish, it says "Do you think this is sensible?" like our usual speeches. Beside it, people know that why they cannot kill people unjustly or sex with their mothers. As logic progresses over information, it can go backwards over information in the other name go into ignorance as well. A basketball player who can basket from midfield and whose skills increased because of own experience can calculate the score according to own logic. If the player is master, maybe can score without looking at the basket. If you want jump of the player from a balcony over a fence that is lower than the balcony and farther, then even if the player can achieve to jump over the fence, maybe he saves his somewhere; but as made complex, also may leave on the fence some of own mind.

For these reasons, human is the best creature of God. Maybe not you; but human is the favorite of God.
We have indeed created human in the best of molds, after we abase them the lowest of the low.

\section{Quran / At Tin - 4,5}

verse talks about it. Human is remaining of God. The situation is like this, that a person asks to God like "Can you create a copy of you?", and then God smiles for the question, and tries, and says, that "I could create this solely..." for the person who asks like he said

Are you the difficult in creation or the sky? He made it.

\section{Quran / Al Nazi'at - 27}

"; but I can create some hammers for them; because they talk without thinking like they are the owners of all the absolute exclusive qualities from past to future. They need time for all works, is it so hard to understand this?"

Furthermore, God can only create his creatures by some potential differences which have to be assumed like matter, particle, and any type of sensed different mass even for souls. Saying "God is able to create the creatures without any reason, cause, instrument." is completely relative. He does not create like us. For example, if you want to teleport to somewhere, you have to pray or you have to make some machines, and also you realize all the act by God's creation; but God only thinks when he wanted to create something without an instrument which is similar to our instruments, reasons; he is not at our situation, condition even if he uses the same space. This reasons for our understandings, and there is an absolute red line even if he creates by some particles.

Mary said that o my Lord, how shall I have a son when no man has been touched me? He said that such, God creates what he desired. If he decides for a work to be of it, he only says 'be', and it just happens.

\section{Quran / Al 'Imran - 47}

verse talks about it; so at this point, Charles Darwin who is the owner of the famous evolution theory [10] maybe did a masterful shot to the target board when even we had not been known anything yet about DNA. As actually God creates by some matter even if his creation is different than our usual ones, he creates them by physical frames with a frequency and the same with computer logic as relativity and uncertainty warned. This can be the same for looked for or sensed situation, condition and it can be said even another situation is not possible.

Did not the unbelievers see that when the sky and the earth were close we uncoupled them, and created all the living things from water.

\section{Quran / Al-Anbiya - 30}

verse may be assumed as an evidence for this. Maybe we have more water than lifeless objects; because we were in the sea in the past, and used it to be formed by God like it has been oneself.

A long time passed during human has not been a known thing.

\section{Quran / Al-Insan - 1}

verse can be assumed as an evidence for God's creation like self-creation over time. Maybe not human; but other lives may be have been created by this way, by an instrument, reason. Additionally, there is no difference between any organs. 
They are the same in the computer logic; thus Darwin's methods may be misleading, and the situation may be hyper irrelevant easily as well. Because of this reasons, we must use super computers to understand the formation with any combination.

God grew you from ground like vegetation.

Quran / Noah - 17

and

Whereas he created you by some stages.

Quran / Noah - 14

and

He is who made nice creation of everything, and started to create human from mud.

Quran / As Sajda - 7

verses can be some evidences for them as well.

Adam was created by an extract which was filtered from mud.

Quran / Al Muminun - 12

verse can be an evidence for DNA or DNA creator temporary organ as a basic seed which was created as an extract formed by, from mud; thus water and some the main life elements, and these elements do not have to be carbon based. Maybe we are; but there can be another creatures which had been created by another creation I think.

\section{Conclusion and Recommendation}

When we started a work which its results are uncertain as there is no more choice chance, there cannot be chance of finding the simplest, the cheapest, the most quality and timesaver one always. On the other hand there are positive economic, technological and administrative discoveries which we must apply them to our life as soon as possible; but in the information stated the above, some simple positive changes that is looked for and interest us show themselves.

\subsection{Energy}

Energy is the basic requirement for the continuation of life as it keeps existence standing. There is no problem where energy is not problem there. Energy producers that produce their own energies and also provide energy for external devices freely, are possible as more clearly is not possible as well according to matter's structure and nature.

Electricity transmission lines can be removed in the earth. They can be removed by the air vehicles which produce their own energies, land and take off vertical manner, and by the workers which have a back thrust engine which was told about the engine in 6.2. Each day, these lines will grow as population grows; thus instead of these lines, various personal energy generators must be manufactured. For buildings or houses, the needed energy generators can be produced by some cheapest methods and by some needed economics transformations; by the way, the producers can be produced freely. By an amount of money like 5 billion dollars, a state which has 100 million people will not need energy forever by big enough self-running personal generators, heating is included to the energy as well for onetime costs for generator materials.

Land and air vehicles, and all of another transportation vehicles can produce their own energies; so fuel in the other name energy will not be a problem anymore forever. We do not need nuclear power plants. An accident is the accident because of some unknown and unpredictable mistakes which they are not done willingly, and when it occurred, it does not mean anything.

\subsection{Space}

New habitats and discovery in their place of new heavenly bodies interstellar or in the sun system are too expensive by our technology in our hands; so some simple methods must be developed. As energy is not a problem, some techniques can be developed which transform energy into thrust. Energy is not independent of mass and thus force. They are convertible for each other.

$$
m v^{2} \sin \alpha / r(K g s)
$$

centrifugal force is a powerful closed system thrust engine potential independent of mass of air or water without opposite torque, and for example, can be consist of tilting of a propeller blade. Propellers always turn at the same speed. I came up with this engine, when I was thinking about electrical charges could gain power by an energy reduction same as happening in this engine. Much more acceleration could obtain than Jet Fighters in the same volume without fuel or other energy resources. For braking, the pressure occurred as a result of sliding the propeller upon the center revolving, could be used by giving directions in both directions to the blades. Also a wall in front of the propeller can be used for brake to push the blades back by using propeller's sliding momentum. It can work everlasting by producing its own energy with electricity energy. It can be used for asteroid mining, combat the asteroids threatening the world, the deepest plows that the engine is placed on center of a triangle and the plows are at the corners, space explorer robots with instant communication apparatus with a simple electronic calculator program, sending radioactive wastes to the sun, space vehicles, water ice shipment in the colonies, speed of light and length contraction experiments against possibility of change state of inertia and going faster than light of different heavenly bodies which have different masses, and astronauts' back thrust engine. With the world's gravity acceleration, it is possible to go to Mars, when Mars is at uttermost distant within 1 week manned and at the same day unmanned with changing accelerations. Also to Alpha C. in 10 years manned manner and to some habitable planets in 30 years. We need an international riding big like a stadium maybe has an electromagnetic plasma shield that particles turns at the required distance from the body at high speed and collide with all the space stuff, evaporate them. Also must have a centrifugal force toilet because of head-shot requirement. Lifting is not problem. There are many habitable planets in 20-25 light years; but there is a problem. We need real mass effect that means gravity; because all the particles we have must be attracted one by one. It is required by our metabolic pressure balance especially by capillaries. It is possible by rotating a mass around itself. Mass effect is multiplied by frequency as required by uncertainty; but we need incredible masses and speeds which are almost impossible. Also a gravitational torque will occur that means mass impacts at a frequency may separate or provoke matter. This characteristic of matter can be used for example moons' locality status; because this mass effect that caused by axial rotation of planets effect the gravity. We can calculate the distance between the moon and the earth and their radius and earth shapes by high technology devices and compare them according to Newton's classic mechanics gravitation formula, and it will say us the moon is local that was a part of the earth or imprisoned external heavenly body by induction and reduction methods. Also this affects the satellites.

Matter is blank and doing every single task in a time period, for genesis and the tasks, which are relatively to the perception in space, a part of the mass will left behind during 
the movement and will gain on itself with a situation such as a super position. This must cause of burning itself out. This feature of the substance, originating from uncertainty, could be used with the intent of communicating with distant lands, faster than speed of light travelling radar, mass transfer part by part, jump ships "Locusts" which all of the particles of the space ship vibrates all together in a resonance, energy producer is included as well with some time differences, and monitoring distant lands by disruptive telescopes corrected through computer. If a particle is sent by a huge energy in the smallest time, it can be assumed at the same time at two places, and can be taken back. In the jumping period, there is caused a mass effect as $e / c^{2}(K g s)$; thus a jump which will be done around the earth would slide our world not only the earth, jumping time is also important in that situation, and the energy would completely ejaculate in an unwanted mistake as well. There would be needed too much energy for jumping; but at least some methods could develop for communication.

This method is a bit cutting or coding method and will be needed less energy or recording area at the same amount of the cutting. It can be also used for sound, display or all other electronic needs. I found this method while thinking on prime numbers and $\mathrm{P}=\mathrm{NP}$ equation. Even if a $\mathrm{P}=\mathrm{NP}$ situation is not possible, gave me the thing I want by being a muse. $\mathrm{P}=$ NP situation and bit cutting method is like the below.

\section{A shot break for Math in Physics}

All prime numbers except 2 are odd whole numbers which are not divided by any whole number except by themselves and 1 on condition that the result is a whole number, and they exist in an infinite number.

\section{Assumption 1}

Table 1: Non-prime odd numbers

\begin{tabular}{c|c|c|c|c|}
$\mathrm{f}(\mathrm{n})$ & $6 \mathrm{n}+3$ & $10 \mathrm{n}+5$ & $14 \mathrm{n}+7$ & $18 \mathrm{n}+9$ \\
\hline $6 \mathrm{n}+3$ & $\mathbf{3 x 3}$ & $5 \times 3$ & $7 \times 3$ & $9 \times 3$ \\
\hline $10 \mathrm{n}+5$ & $3 \times 5$ & $\mathbf{5 x 5}$ & $7 \times 5$ & $9 \times 5$ \\
\hline $14 n+7$ & $3 \times 7$ & $5 \times 7$ & $\mathbf{7 x 7}$ & $9 \times 7$ \\
\hline $18 n+9$ & $3 \times 9$ & $5 \times 9$ & $7 \times 9$ & $\mathbf{9 x 9}$
\end{tabular}

All of the odd numbers which are not prime numbers are the numbers which have at least two multipliers whether prime or not and multiply in a way dependent on $f(x, y)=$ $(2 x+1)(2 y+1)$ function whose variants are within the range of $[1, \infty)$. To organize and demonstrate it in a table, the table will be like Table 1 .

The same rule is existent both for the horizontal and the vertical and all of the positive odd whole numbers in the table are the numbers which can definitely be divided by at least any odd whole number; thus the odd whole numbers which are not included in here are only the prime numbers. If prime numbers were finite, for

$$
\sum_{x=1}^{\infty} \sum_{y=1}^{\infty}\left(\frac{2 x+1}{(2 x+1)(2 y+1)}-1\right)
$$

the result of the total operation of

$$
\sum_{y=1}^{\infty}-\frac{2 y}{2 y+1}
$$

would be 0 for each y value; however this operation never converges, and each result is different; thus it can be maintained that

Prime numbers are infinitely many numbers together with the odd numbers which can be divided by another odd number.

\section{Proposition 32}

Now that the prime numbers are infinitely many numbers so the number of prime numbers for any range determined, which odd whole number the $n$. prime number is, the prime factor of any number determined, its multipliers or number of the prime factors and which odd whole numbers these multipliers are can be found out.

\section{Assumption 2}

To find out in which column a positive odd whole number determined was lastly it must be ascertained that in which perfect square's column it was lastly, that it was in main lines or line spaces. Square zone of sphere is a reference for elimination. It is not compulsory to base on the first number in each column

For $b=[1, \infty)$, the perfect square of each $\mathrm{a}=2 \mathrm{~b}-1$ positive odd whole number is separated like $a^{2}=1+4 b(b-1)$; thus to find out which nearest-to-base positive odd whole number's square a positive odd whole number determined must be, it is required to create a function which rounds down any fractional part by placing whole value. For any $\mathrm{x} / \mathrm{y}$ operation based on the simple division rule as it is $y n+m=x$ for $r=x-y n-$ $\mathrm{m}$ the sum of

$$
\lfloor x / y\rfloor=\sum_{n=1}^{x-y}\left(\sum_{m=0}^{x-y} \frac{1-(-1)^{2^{2^{2}}}}{2}\right)
$$

can be used. Which nearest-to-base positive odd whole number's square a v positive odd whole number determined based on this greatest integer function must be can be found out based on this greatest integer function for $r=v-1-4 n(n-$ $1)-m$. The result a an s number and provides the maximum number of columns which will be studied for the number determined and $2 s+1$ number provides the basic odd number composing the last column.

The number of columns yielded from the perfect square elimination being $\mathrm{n}=[1, \mathrm{~s}] \mathbf{2 n}+\mathbf{1}$ numbers are adequate for controlling whether v positive odd whole number is prime or not when divided by $\mathrm{v}$ positive odd whole number; thus it will be adequate to perform s numbers operation to control whether a positive odd whole number is prime or not.

\section{Assumption 3}

If both of the first two multipliers were the last column found for the $\mathrm{v}$ positive odd whole number determined for its elimination of black belt; s being the last column or the number of the columns, the number determined could only be $(2 s+1)^{2}$. This situation is only possible if the number determined is a perfect square. If the first two multipliers were in any column or columns after the last column; s being the 
number of columns, the minimum number which could be formed must be $(2 s+2)^{2}$. This is a bigger number than the number determined itself; thus at least one of the multipliers are only in the s column or in the columns before $s^{\text {th }}$ column.

As it is not possible to control whether an even number are prime a mathematical interpretation can be done to control whether $\mathrm{v}$ positive odd whole number is prime. When it is $\mathrm{d}=0$ for $d=v-(2 a+1)\lfloor v /(2 a+1)\rfloor$ whole value element, $2 \mathrm{a}+1$ positive odd whole number is aliquot divisor of the $\mathrm{v}$ positive odd whole number ; thus $\mathrm{v}$ is not prime. In other situations it is not prime and when $2 \mathrm{a}+1$ divisor is tested within $\mathrm{a}=[1, \mathrm{~s}]$ range $\mathrm{v}$ is prime if the status of $d \geq 1$ is conserved; thus

$$
\sum_{a=1}^{s} \frac{v\left(1+(-1)^{2^{d}}\right)}{2}
$$

operation provides $\mathrm{v}$ number if $\mathrm{v}$ number is prime and if not the result is 0 .

If it is $\mathrm{v}=2 \mathrm{x}+1$ and $d=\sum_{a=1}^{x-1} 2 x+1-(2 a+1)\lfloor v /(2 a+1)\rfloor$, the number of prime numbers between $2-\mathrm{v}$ can be found based on

$$
2+\sum_{x=2}^{(v-1) / 2} \frac{1+(-1)^{2^{d}}}{2}
$$

function .If it is $d=0$ for an a value $2 x+1$ is not prime. In other situation it is newly prime. It can be turned also for the number of prime numbers within any range .For the $n$. prime number within any range if it is

$$
m=n-2-\sum_{x=2}^{y} \frac{1+(-1)^{2^{d}}}{2}
$$

based on the same function

$$
p=\sum_{y=2}^{\infty} \frac{y\left(1-(-1)^{2^{m^{2}}}\right)}{2}
$$

operation provides $\mathrm{p}$ which is the $\mathrm{n}$. prime.

The odd numbers stated till the main number of the last column will not be adequate for the operation of finding prime factor;because this operation necessary for finding out one of the prime factors and the other multiplier can be evident after the last column; thus, to find out the number of prime factor, all exponents of the main numbers in the columns must be divided by the number determined in a way it does not exceed the number determined and controlled.

$\mathrm{d}$ operation for full value of $v /(2 a+1)^{n}$, greatest integer function must be $d=v-(2 a+1)^{n}\left\lfloor v /(2 a+1)^{n}\right\rfloor$; thus the sum of

$$
t=v-\frac{v}{(2 a+1)^{\sum_{n=1}^{\infty} \frac{1-(-1)^{2}}{2}}}
$$

calculates how many exponents of $2 \mathrm{a}+1$ numbers for each a value are aliquot divisors of $\mathrm{v}$ for each number determined. Then when $v$ is divided what is left is the prime factor itself and when it is subtracted from $v$ again if the result is $02 a+1$ number is not a multiplier. If the result is bigger than 0 it is a prime factor; thus

$$
\sum_{a=1}^{s} \frac{1+(-1)^{2^{t}}}{2}
$$

function provides the number of prime factor for $\mathrm{v}$ which is the positive odd whole number determined. If the result is still $0, \mathrm{v}$ is a prime number.
This is not an evidence to the impossible status of $p=n p$; however as all prime numbers are certainly odd number and between two consecutive odd numbers which are the multiples of 3 , it can be assumed that only $6 x+3$ rule and so multiplies of 3 are in the set of odd numbers and the odd numbers in-between can be assumed as prime. This increases the number of prime numbers and decreases the possibility of the multipliers to be odd whole number which can be divided and so the number of non-prime factors. Then if prime numbers are assumed in the place of the multiplies of 3 and the numbers in-between positive odd whole numbers which can be divided, the separation rule of primes would be $(x-3) / 6$ and the numbers in-between would not be important; because there is now a function providing separation of primes and it is now known where are prime factors sought. The result of this equation would be checked to understand whether a number is prime or not when this has happened. If the result is a whole number, then the number is prime and if not, it is not prime. If the number is not a prime at least $\lfloor(x-3) / 6\rfloor$ operations would have to be done to find out prime factors; thus a status of $p=n p$ is not possible; thus it can be maintained that

The state of $\mathrm{P}=\mathrm{NP}$ is only a dream.

Proposition 33

and this is the proof of the status of $p=n p$ based on prime numbers. Only 1 evidence is enough. I am of the opinion that this is the certain evidence. When it is accepted as true it will ease the minds of mathematicians and the companies in need of security to some extent. Even if quantum computing is not available absolute protection is possible in a time interval if security is reduced to decryption. A computer whose velocity increases infinitely becomes uncertain relative to protected computer at a point; the range of trial with algorithms for uniform velocity must be diminished, which is not possible.

For the floor function also a whole value function which provides a whole number result can be created by using only the rule of dividend by 2 . For each $2 x+1$ odd number there is a whole value once in a $2 \mathrm{x}$ number between the whole values; thus it is also related to the separation of primes. A compound function can be written together with the separation of primes.

Additionally as it can be done a ratio test with the rule on the Table 1 for Goldbach's conjecture [11] according to Euler's answer, actually it can be built a simple logic for it. I remembered it and the others the below during the revision of the article, and as I solved their infrastructure in a few hours lucky manner, I added as well. If the given positive even number is $2 \mathrm{x}$, then use Table 2 the below for $2 \mathrm{x}-3$ odd number as the upper limit. The left column and the other always occur reverse aligned manner.

Table 2: Reverse aligned odd numbers

\begin{tabular}{|c|c|}
\hline 3 & $\cdot$ \\
\hline 5 & $\cdot$ \\
\hline 7 & $\mathbf{1 3}$ \\
\hline 9 & $\mathbf{1 1}$ \\
\hline 11 & $\mathbf{9}$ \\
\hline 13 & $\mathbf{7}$ \\
\hline$\cdot$ & $\mathbf{5}$ \\
\hline$\cdot$ & $\mathbf{3}$ \\
\hline
\end{tabular}


Always, both of the columns occur as positive odd numbers which are non-prime as the left column is always formed with non-prime numbers.

\section{Assumption 1}

The left column and thus the right column always contain consecutive positive odd numbers that 3 is the first number of them; thus none of the two columns contain only non-prime numbers.

Always, both of the columns occur as positive odd numbers which are prime as the left column is always formed with non-prime numbers.

\section{Assumption 2}

The left column and thus the right column always contain consecutive positive odd numbers as stated in the first analysis that 3 is the first number of them; thus none of the two columns contain only prime numbers.

If this is the situation, then positive even numbers never be formed by only prime numbers or only non-prime odd numbers; thus a positive even number always be formed by least two prime numbers and one prime plus one non-prime number if you add 1 as well and ignore first two situation which are 2 and 4 as there is no other number and thus choice in Table 2 for these numbers.

Over this method, it can be said, that twin primes are infinitely many numbers. If you make infinite number of table groups the same as Table 2 rule for all $2 x-3$ numbers respectively multiples of 3 which are non-prime odd numbers and occur after two consecutive odd numbers according to $6 x+3$, then there will be always some groups that these multiples of 3 form the given even numbers at the center of the table as multiples of 3 which are non-prime odd numbers and occur after two consecutive odd numbers. The other groups always occur between these multiples of 3 as well and then again a table which has two the same multiples of 3 at the center occurs. If this is the situation, then we have only 3 choices. After this 3 choice again the table which has two the same multiples of 3 at the center occurs.

For the other conditions,

Always, there will be non-prime odd numbers between two consecutive multiples of 3 .

\section{Assumption 1}

If there are always two non-prime numbers between two consecutive multiples of 3 , then all the table contains nonprime odd number. The table can only formed by positive odd numbers, and odd numbers are not formed only by non-prime numbers.

Always, there will be prime odd numbers between two consecutive multiples of 3 .

\section{Assumption 2}

If there are always two prime numbers between two odd consecutive multiples of 3 , then it must be formed all the table by multiples of 3 as non-prime numbers. This is not possible as there are infinite numbers of non-prime numbers different than multiples of 3 .

If this is the condition, always there must be twin prime numbers which form the given even number $2 \mathrm{x}$ at the center of the table two times for each table in some tables forever together with prime plus non-prime groups; because when multiples of 3 is at the center, the difference is 4 between the numbers which are the closest to the center in two column reciprocally as the difference of two odd numbers which have one odd number between them is 4 . In the other situation, the difference is 2 at the center back to back directly. Whereby analysis of assumption 2 says that there are not only prime numbers between multiplies of 3 and also as analysis of assumption 1 warned there are not only non-prime numbers, and finally if the difference 2 at the center in back to back twin group, there must be twin primes forever by some intervals.

Mersenne primes which are depended on

$$
2^{p_{1}}-1=p_{2}
$$

function does not give always prime result. If we form the function as

$$
2=\left(p_{2}+1\right)^{\frac{1}{p_{1}}}
$$

then $\left(p_{2}+1\right)$ always must be a number like $2^{n}$. If this is the situation, then for $p_{2}=2^{n}-1$ for some $n \geq 1$ positive integers the result always must be prime number.

$2^{n}-1$ is always a positive odd number and is always prime number.

\section{Assumption 1}

For example let us use a function like $10 x+3$. If there are infinite numbers of intersection points with $10 x+3$, then $2^{n}-1$ is not always a prime number.

For $2^{n}-1=10 x+3$ it must be

$$
x=\frac{2^{n-1}-2}{5}
$$

and for some $n>2$ positive integers forever, this function gives $\mathrm{x}$ integers forever as 5 or multiples of 5 when $2^{n-1}$ had 2 in the first digit as $2^{n-1}$ has a constant repeat as $2^{n-1}$ can only formed by 2 . If this is the situation, this explains that Mersenne prime function does not give always prime results for the given variable primes.

$$
\begin{aligned}
& 2^{n}+1 \text { is always a positive odd number and is } \\
& \text { always prime number. }
\end{aligned}
$$

\section{Assumption 2}

In the same manner, let us use a function like $10 x+5$. For $2^{n}+1=10 x+5$ it must be

$$
x=\frac{2^{n}-2}{5}
$$

and this has the same result with (72); so it can be said that Fermat prime function which is dependent of

$$
p=2^{2^{m}}+1
$$

and thus for $2^{2^{m}}=2^{k}$ of $2^{k}+1$ does not give primes always. As the result, as Mersenne and Fermat prime functions don't give prime result always, they give sometimes prime results forever. $6 x+5$ function is always between two positive odd multiples of 3 for positive $x$ numbers and is always the next one. For $2^{a}-1=6 x+5$ it will be

$$
x=\frac{2^{a-1}}{3}+1
$$

and as $2^{a-1}$ is only composed of 2 it is not dividable by 3 ; so $\mathrm{x}$ on (75) is always decimal and $2^{a}-1$ can never exist as the next odd number after 3 . Also as non-prime numbers or prime numbers cannot only exist alone as the previous odd number just before of 3 or after 3 as the next one consecutively as we can see on (77) and (78), Mersenne function always must give some prime results forever as well. $6 x+7$ function is always between two positive odd multiples of 3 for positive $\mathrm{x}$ numbers and is always the previous one. For $2^{b}+1=6 x+7$ 
it will be

$$
x=\frac{2^{b-1}}{3}+1
$$

and as $2^{b-1}$ is only composed of 2 it is not dividable by 3; so $\mathrm{x}$ on (76) is always decimal and $2^{b}+1$ can never exist as the previous odd number before 3 . Also as non-prime numbers or prime numbers cannot only exist alone as the previous odd number just before of 3 or after 3 as the next one consecutively as we can see on (77) and (78), Fermat function always must give some prime results forever as well.

Additionally, if all the positive odd numbers before of odd multiples of 3 are non-prime numbers then as all positive odd numbers are between two consecutive odd multiples of 3 and there are two odd numbers between them, all the positive odd numbers after of odd multiples of 3 have to be prime numbers; but it is not possible as prime numbers cannot have a first-degree function because of divisibility condition which is caused by constant intervals even prime numbers have a certain separation. For example by using positive integers for between two consecutive odd multiples of $3,6 x+5$ function is always between these two odd multiples of 3 and is always the next one. If we use separation of 5 then for $6 x+5=10 y$ -5 it will be

$$
y=\frac{3 x}{5}+1
$$

For positive integer $\mathrm{u}$ numbers and $\mathrm{x}=5 \mathrm{u}, \mathrm{x}$ on (77) shall always form y as an integer; thus there are always some positive odd multiples of 5 at the next positions of positive odd multiples of 3 . For the second and last situation, $6 x+7$ gives the previous odd numbers of odd multiples of 3 . If this is the situation then by using $6 x+7=14 x-7$ equation as odd multiples of 7 is dependent of $14 y-7$ function, it will be

$$
y=\frac{3 x}{7}+1
$$

For positive integer $\mathrm{u}$ numbers and $\mathrm{x}=7 \mathrm{u}, \mathrm{x}$ on (78) shall always form y as an integer; thus there are always some positive odd multiples of 7 at the previous positions of positive odd multiples of 3 . If this is the situation then there are infinite numbers of palindromic primes by the rule of odd multiple of 3 which is $500 \ldots 007$ has variable number of zeroes between 5 and 7 , and as prime 5 and 5 .

As Collatz's problem,

If there were an odd number which cause an infinite loop by the Collatz's rule by transforming to the other odd numbers, and cannot be decreased until 1 forever, then this number $\mathrm{x}$ cannot be $2 \mathrm{n}$ for $3 \mathrm{x}+1$ function as it will be a $3 x_{n+1}+1$ even number because of $x_{n+1}=\left(3 x_{n}+1\right) / 2$ or $x_{m}=\left(3 x_{n}+1\right) / 2$ always or $\mathrm{x}$ goes to 1 , as multiplying an odd number by 3 means making this number the number which is dividable by 3 ; thus if you add 1 to $3 \mathrm{x}$, it shall be an even number and it shall appear always after odd multiples of 3 and is the next one, first even number just after odd multiples of 3 by $6 y-2$ rule as there are always two consecutive odd or even numbers between two consecutive odd multiples of 3 .

\section{Assumption 1} be

We can use $2^{n}=3 x+1$ equation. For this equation, it will

$$
x=\frac{2^{n}-1}{3}
$$

and as $2^{n} /(3 n+1)$ ratio always increase, there must be other $2^{m}$ numbers. As there are always 3 even numbers between 2 consecutive odd multiples of 3 , for $3 y+3$ numbers which are at the middle always between two consecutive odd multiples of 3 the result of $\left(2^{t}-3\right) / 3=x$ will always be

$$
x=\frac{2^{t}}{3}+1
$$

and as $2^{t} / 3$ is always decimal as $2^{t}$ is only formed by $2, \mathrm{x}$ will always be a decimal and it can be said that a number like $2^{t}$ cannot exist at the middle between two consecutive odd multiples of 3 for any $t>1$ integer. For the numbers which occur before odd multiples of 3 and are at the final place between two consecutive odd multiples of 3 being $2^{r}=$ $3 x+5$, it will be

$$
x=\frac{2^{r}-5}{3}
$$

and then all the $2^{n}$ numbers are either the next or the previous one for consecutive odd multiples of 3 ; but maybe (79) or (81) can occupy alone both of these places, and maybe one of them does not have any intersection with these points. For $\left(2^{r}-5\right) / 3=\left(2^{n}-1\right) / 3$ equation, it will be

$$
2^{r-2}+2^{n-2}=1
$$

And it can be said that this equation is valid for only 1 value for $n=2$ and at the same time for $r=3$. For the other values as the functions are formed by 2 , the difference has to be 0 or least 2 . If this is the situation, it can be said that (79) and (81) never intersect, and they have always some $\mathrm{n}$ and $\mathrm{r}$ positive integers forever; so $2^{n}$ is always just after and $2^{r}$ is always just before of odd multiplies of 3 for some $n$ and $r$ values. As summary, (79) and (81) have infinite x odd results; so either $\mathrm{x}$ goes to 1 directly by $2^{n}=3 x+1$ even number or visits a $x_{n+1}=\left(3 x_{n}+1\right) / 2$ or $x_{m}=\left(3 x_{n}+1\right) / 2$ conditions and goes to 1 again as there is no alternative.

As there are always some intersection points, running away from to be $2^{n}$ numbers is not possible. There is no way to do this; thus if we work by using positive integers, the numbers which can be obtained always as 1 by only dividing by 2 can be expressed by like a $y=2^{n}$ function. As $2^{n}$ is not dividable by odd numbers, being $\mathrm{x}$ is a positive integer, for $x_{0}=x$ and for

$$
\begin{gathered}
y_{n}=\frac{\left(3+\frac{1}{x_{n}+\frac{1-(-1)^{2^{x_{n}}}}{2}}\right)^{\frac{1-(-1)^{x_{n}}}{2}}}{2^{\frac{1+(-1)^{x_{n}}}{2}}} \\
p_{n}=\sum_{m=1}^{\infty}\left(\sum_{k=1}^{\infty} \frac{1-(-1)^{2^{\left(y_{n}-2^{m} k\right)^{2}}}}{2}\right) \\
x_{n+1}=\frac{y_{n}}{2^{p_{n}}}\left(\frac{1+(-1)^{2^{\left(x_{n}-1\right)^{2}}}}{2}\right)
\end{gathered}
$$

elements, Collatz's rule will be as

$$
1=\sum_{n=0}^{\infty} \frac{x_{n+1}\left(1-(-1)^{2^{\left(x_{n+1}-1\right)^{2}}}\right)}{2}
$$

and it is the most stupid way of writing 1, that belongs to me. Also any partial function can be written like these methods similar manner as a calculus function.

As $2^{n}$ numbers are always just before or after of multiples of $3,2^{n}+1$ shall always be dividable by 3 when $2^{n}$ is the previous one for some $n$ integers as $2^{n}$ is not always only before or after as we can see at (79) and (81) proofs. If this is the 
situation, being $\mathrm{p}$ is a prime number, for $x^{2}+1=p$ equation $\mathrm{p}$ can only exist before or after odd multiples of 3 . For just after it will be $6 y-1$ and for just before will be $6 y+1$ for $y>0$. For $x^{2}+1=6 y+1$ it will be $x=\sqrt{6 y}$ and we can use separation of $2^{n}$ numbers. For $2^{n}=6 y$ there shall never be an intersection point as it will be $2^{n-1}=3 y$ as $2^{n-1}$ can only be formed by 2 . For $x^{2}+1=6 y-1$ it will be $x=\sqrt{6 y-2}$ and we can use separation of $2^{n}$ numbers again. The equation will be $x=\left(2^{n}+1\right) / 3$ and as $2^{n}$ numbers are always just before or after of multiples of 3 and are not always only before or after as we can see at (79) and (81) proofs for some $n>1$ integers, and already if you add +1 to $2^{n}$ it will be equal to odd multiples of 3 and can be divided by 3 as well when $2^{n}$ is just before odd multiples of 3; thus it can be said that there are always some prime numbers just after a perfect square as non-primes or primes cannot exist only before or after odd multiples of 3 as stated by (77) and (78).

Return of 6.2

The natural vibration of matter on surface caused by its own emergence is a processor potential. Also a high quality processor with a processing capacity of $10^{15-20}$ Hertz at the size of a coin can be made with the vibration emerging from the situation that the energy absorbed by many particles, which revolve around in the magnetic field, in sequence from the nanometric electrodes placed around the circular field changes the flow on the materials which are stable at a place in the same magnetic field maybe even by using insulators. A serial production can be carried out by sending ions to magnetic plates by the way of scanning electron microscope or CRTs. If the tried ones do not work, the system makes the processor ionized and uses the particles again as well.

These processors can be used to cut data bits. Either solely 0 or solely 1 of the bit groups recorded in a re-writable disc must be recorded according to their first or $n^{\text {th }}$ degree remoteness among themselves throughout data considering which one is less or which one can be compacted better with smaller degree equations. For example, for an information stated in Table 3 and Table 4 the below,

Table 3: The current data bits to be cut

\begin{tabular}{c|c|c|c|c|c|c|c|c|c|c} 
& 1 & 2 & 3 & 4 & 5 & 6 & 7 & 8 & 9 & 10 \\
\hline $\mathrm{A}$ & 0 & 1 & 0 & 0 & 1 & 1 & 0 & 1 & 1 & 0
\end{tabular}

Table 4: The marked bits to be cut

\begin{tabular}{l|l|l|l|l|l|l|l|l|l|l} 
& 1 & 2 & 3 & 4 & 5 & 6 & 7 & 8 & 9 & 10 \\
\hline $\mathrm{A}$ & $\mathbf{0}$ & 1 & 0 & $\mathbf{0}$ & 1 & 1 & $\mathbf{0}$ & 1 & 1 & $\mathbf{0}$
\end{tabular}

the zeroes until $A 10$ line must be excluded according to $f(x)=3 x-2$ function. This information is recorded as A124 and means that there are 4 times 0 which the distance is two bits between each of them, and are existent for $A 1$ starting address. The existence of capitals and lower cases side-by-side or with a distance between them has different meanings and these are compacted constantly. Even if transfer was at the level of $K B$, the compressed data would able to be at the level of $P B$ according to compaction. This can be used for virtual full body complication machines which all the physical and chemical properties of the particles and the dynamic conditions which are caused by the atmosphere are installed; high speed camera, speech apparatus analyzing brain waves by being stimulated by the waves, which are kept on border-line and multiply voltage difference not producing current when it is not active; simulations of universe; materials science and also for mathematics. Also we can use the computers for DNA research for real Jurassic Parks. Maybe as the first work, we can create a live mutant uterine by using a suitable animal, according to the research results. This method could also be used for software. The number of showed pictures each second in games is certain even if they are variable. Supercomputers do the calculation of games and then encode the game. A very old game and the newest one work at the same difficulty. PCs only do work of erasing and opening the bits. Just some codes which give to the pixels voltage orders.

There is no threshold value and matter stores energy; thus the condition can be used for space collecting on particles by the time differences between the processor's conductor, semiconductor or dielectric nano-electrodes that each one vibrates at $10^{10-15}$ Hertz and maybe more according to the used materials. Also the times when the universe is not there can be used; because the universe moves circular for its each point. A particle in a great intensified magnetic field, could be beamed up automatically when the time difference sum is taken over the threshold value due to natural vibration of the magnetic field's itself. Maybe Magnetars produce the high energy particles which are different than production of exploded stars, and finally they lose their strong magnetic field because of the particle loss, evaporation and frictions.

Current passenger planes must be history, like space launching pads must be. The new wingless flat passenger planes will be extremely safe due to not having an explosion risk; because they do not use fuel. They have the closed system engine, and can move vertically. Some small thrust engines which are produced by the same method on the seats can save the passengers at the required height for ejection. Tranquilizer gas can be used before ejection. Also it can be built with a guardian armor against air pirates and thus guns. Even it can be experienced a conflict in the plane. The weight is not so important as much as before; thus it can be done, and also maybe a big plane parachute is prepared for plane itself, it works by hand for the worst possibility. Maybe we should build it against radiation as well to go from higher place to open place for example personal cargo planes, helicopters etc. as well. Some of them can go supersonic or even hyper-sonic from the space. Also free space-induced turbulence is not possible with these engines; thus maybe we should skip the windows as well for more comfortable, healthy travels. Maybe we can use a thin vacuum layer against sounds as well, the engine is included, and the vacuum is controlled and refreshed by some small engines. Airports can be built as circular high buildings by some plane area stairs with different radius. Every plane's place is a small circular area around the circular building on the stairs as well in these airports. We build a very high tower at the center of the building and they can see anything by naked eye.

New fixed non-orbital satellites can be placed at a certain level of height with a standard compulsory bit cutting apparatus for global communication. It will be cheaper and cheaper; thus maybe we can produce its some the same backups at the same time, and we can send it in a half hour if there will be a problem. By this way, spy satellites cannot wander around, and it is quite fair. Special works can be done by some cheap non-orbital satellites or need special permission. Low EM wave energy and more data. 
Space debris can be cleaned by some vehicles like scorpions that there are millions of high energy lasers between the hands in a high volume area. Maybe magnetic plasma or water can be used as well.

Cargo companies should use some unmanned or manned small low altitude air vehicles fleet that has certain route against passenger planes for instant transport in the name of high quality service. If the customer has the money, then it means there is no problem.

The engine also can be used for land vehicles. The vehicles which manufactured with the engine can go on ceiling; because the same method can be used produce land pressure especially for trucks. Heavy vehicles are more affected from centrifugal force because of $m v^{2} / r$, and easily can be displaced and turned-down, that truckers usually feel as they use tanks which cannot be affected from anything. A system always checks the condition, and if it is required then the system controls the arms by changing the radius and the angles independently of one another. Car heating and cooling works can be made by thermoelectric semiconductors instantly in the vehicle. The same engine can be used as an advanced brake system. Classic brake systems cannot allow vehicles to stop instantly, because even if the brake system is ABS, the vehicles will slide like the boards on snow. If an accident occurs, the land pressure system is automatically activated and it applies a huge force at the opposite direction of the accident by big but not fatal $\mathrm{G}$ forces that occur in a smallish time interval by the main engine or the land pressure system. The land pressure system always runs at required various speeds according to the current car speed and the calculations on the speed. These vehicles also can be used for free space, the Moon and Mars races. Also we can use the engine to save the tourists for the places like Everest with some sensitive heat cameras. We should give to the tourists some electronic wristbands to help, and we should watch them by name and coordinates from a screen in a center by using a non-orbital fixed small Everest satellite over Everest. We can use some strong special prefabricated housings with many bedrooms at some different acclimatization points with no energy requirement there as well; because nobody cares about the body condition. Oxygen and cold are a serious problem as well. For example they should do work of condition, gymnastics and weight at least 6 months before the climbing I think; because 72 times of training is not too many in 6 months. Also they should gain weight a little as fat if they are not already fat, and they must be careful about nutrition against elemental problems against fatal body problems in this period. In the same day, they may say hello to the other world especially by some heart attacks depended on elemental problems. They will see that there are much more difference in terms of climbing if they do training before. The joy should not turn into the pain.

Cargo ships which can only cruises on sea and ocean at a smallish distance from water, high enough can be manufactured. Ships should carry underwater jet pack by the thrust engine and self-running generators for the people on them. Every ship can lift itself by its own current engine; because there is no opposite torque because of air or water. It will be very safe against huge waves and storms. Also we can use high thrust manned or unmanned air vehicles that land and take-off vertically in ports instead of winches and transport land vehicles. We do not need any other vehicle or machine in ports. Fast, cheap and powerful. The air vehicles which do not require external energy will be cheaper than the winches as well. By the way, containers can be high aligned vertically in smaller area.

We can use the same engine for hospitals, for fast medicine and patient transfers.
We should produce some maybe spherical personal space vehicles which generate own energies and thrust instead of the suits which the vehicles provide possibility for discovery and maintenance by some internal vehicle mounted gloves which are maybe robotic arms.

We should manufacture some working machines as powerful air vehicles for earthquake disasters in earthquake first aid stations to remove the rubble faster in a small area to save people. The other machines cannot reach anywhere we want during the rescue effective manner.

We must build a Mars station, and maybe a Venus and a Mercury station. We can build the Mars station movable by some thrust engines that is explained above with radars against asteroids; then we do not need a second assembly and piecemeal transport from the earth and we should make a thin vacuum layer against Mars wind sounds. Also we should build a smoking room; because after this, anybody can travel in space and go to there, and the body condition is not so important as much as before; thus statesmen, soldiers and scientists will go to there astronauts are included as well. The engine which works by centrifugal force stated above stores energy by some transmission parts by force gain to protect the energy which is gained by axial motion. For example, let us assume that a fuel thrust engine means rocket has 1 million kilograms thrust. If you connect it to a ship that is 1 million tones weight, you can't lift it. If you use fuel at amount of the world's weight for years, you can't change anything, because you shouldn't drive a car has square wheels. The total spent fuel could shot the ship like a bullet; but you could not use the energy. If you connect it to a centrifugal force thrust engine you can store the energy; thus force. It doesn't differ for the engine if it is worked or not. If you accelerate a 1 ton object, when it has $1 \mathrm{~km} / \mathrm{s}$ speed, it has a 1 million kilograms momentum per second and then if you connect it to the ship you cannot lift the ship again and your continuation cannot change anything. It slows down. By the values, if you build a propeller that is 1 ton and has $1 \mathrm{~m}$ radius if you can gain nearly $10000 \mathrm{rpm}$ for 1 minute you can gain a centrifugal force that has 1 million tons magnitude without an angle. The second work has $1 \mathrm{~km} / \mathrm{s}$ speed again but it stores energy. We can save all the people with a single space ship in theory. We can save energy. If we can find some asteroids include carbon dioxide, water and oxygen as dry or liquid, we can heat Mars easily.

Lands of the Moon and Mars have to be shared between all of the nations of the earth by draw with divided planet surfaces by pixels. Status of owning land in the earth, population and being useful for science; thus mankind has to be the criterion about sharing the lands. The owned lands in the earth must be negative, and contribution to science and population is positive multiplier.

We must determine a tonnage for asteroids. An asteroid which has smaller tonnage than the determined tonnage cannot be owned by a second company or state; but if the situation is different, infinite number of company and state can work on the same asteroid even if one of them has a bigger work ability than the total of them. Anybody does not have to talk about the mine type; but if it is rare and the company or the state cannot finish the work in a little time then they have to talk about them. Otherwise, they have to work freely for a time because of some penalties. If they brighten us about the rare mines, they can deserve the share at a ratio from all the workers on the asteroid. They can only establish an asteroid research center as well, and play onto the tax if they find something.

Also another thrust engine can be produced. For example, if 3 electrodes are connected on the same line, as distance between electrodes are different and with opposite elec- 
tric fields, and particles are accelerated and slowed between the electrodes then we can get a thrust on a direction according to relative distance of the electrodes and thus amount of the electrical force. The voltage will be same, but because of the distance, $V / d$ ratio will change; thus, the imbalance will create a usable thrust. At the place where the velocity of the particles have zero speed, an electric field perpendicular to the movement direction of the particles can take the particles and feed the system again. This engine is usable in space, but not for more. I do not mean that the engine is bad; because even if the engine is produced for any works, then it will be better than current space engines. Maybe electrons can be used in two coils facing each other for another engine by using the imbalance. Some different imbalances can be produced I think if we think.

\subsection{Vital infrastructure of states}

Ionization in electric field can separate nucleus and electrons of any material as energy is not a problem anymore; so by using mass difference in magnetic field, any material and element can be produced as bullion. This is a very good, simple and the cheapest way of mining and composite material producing additionally by induction ovens, lasers or magnetic plasma boilers which collide the particles and then make them rest in some bullion molds. Energy of an electric field is directly proportional with voltage's square; thus it does not mean for example $1 M W$ energy can separate as much as $1 M J$, and the area is important as well. We can separate them each second at almost $100 \%$ purity. Also we can use vacuum cooling. A system with force efficiency always checks and protects the condition for the cooling.

Silicon is the most abundant element in the earth. Its composite with carbon in the other name silicon carbide is stronger than steel. It can be used for road by some frame methods that some rectangular silicon carbide parts are sit on some silicon carbide rails from some points by intervals perpendicularly, and slide on the rail and sit into the other as the same as battens; for vehicles, other buildings and machines like unbreakable toys durable forever for stress by the required thickness against earthquakes and other negativity. The super ships which have the thrust engine works by free energy, can be produced by using silicon carbide as well. More endurance and lighter. Buildings can be built like matchstick houses by using composite insulators and a simple web with some big near surface earthquake shoes like a skirt as the base, and by the way, disassemble is going to be possible. We need the one-piece unstressable huge shoes; because we should escape the maintenance even this is not possible for very high structures. I know it will be cheaper than cement and steel by some vector frame formations to spread the weight, even if we use much more silicon material. Just a strong skeleton on the ground. The building can be fixed by some winches after earthquakes. Only by onetime spending by the most primitive, simple and rudely methods. It can be built some ionization stations can be named as giga-factories. Building can be built from today to tomorrow when the needed infrastructure was completed. Heating can be done by a helical pipe coil that was heated by electricity, by using house's own air instantly and constantly. Surface heat of the coil can heat the water in a boiler constantly. Just some simple thermodynamics calculations and we need the pipes as thin as possible; because for the same weight matters, if you increase the voltage you can gain more heat for the same energy even if the same amount of the air passes in the pipe. This is almost not a machine and almost there is no engineering. It can be sold by weight. As space is vacuum, it always wants to cool the earth or the other heavenly bodies like the sun. If we use some vacuum pipes, then by using a strong vacuum we can use it for the cooling work. The same method can be used for engine cooling. This can cool the water in the same heating system as well. It is not so hard finding absolute insulators for these temperatures. Whatever, we can find some simple and cheaper ways for all of our works. It does not mean bad. The same devices can be used in space colonies. If they are broken, they can use the replacement, and the new ones can be sent from the earth in 1 day unmanned manner for example for Mars.

Also ionization can be used to produce water from moisture of air or land and sea or ocean. Maybe also we can use heated metals for seas or directly use pressure difference by some self running high frequency pistons. For drinking waters, the water can be fed for minerals by the ionization again. The ionization can be used in space colonies for mine and recycling. In the earth, it can be used for purification of wastewater and industrial wastes. Also maybe for dangerous industrial wastes, we can build some big ionization trucks for the fastest effect in its place.

Our dams must be prepared as huge pools at the some required places against firstly, nuclear attacks and secondly efficiency, danger and more area. Mankind may exit from humanity even about not important subjects. Water can be pumped to the required distances by the some electro-pumps that produce their own energies. The sand is eliminated at the entrance of the dam, and it will be long-lived. It can be built like a cut conic and maybe the sands can be cleaned in the base. The ground soil can be used for high walls against flood. It's not a problem to find more ground from somewhere.

We should build some digger machines for the two poles of the earth. Maybe there are some microorganisms that they do photosynthesis; by the way, we can use them for Mars against the hard weather of Mars. In the south pool of the earth, the height is very high and I think it will be higher because of moisture of the air; thus maybe there are some unknown microorganisms that have not escaped somewhere yet. Also maybe we can find some anti-virus kamikaze bacteria instead of human cells that they attack the other viruses like moribund animal cells in human body or artificial mutant bacteria by neutrons or gamma rays. I think these virus works are very annoying. This is like storing water in a sieve, and the best way is solving the human DNA with all complication combinations, then we can produce some selectively permeable cell polices that work according to installed usual foods. If there is an unknown matter in the entrance, cell destroys the entrance and create a new one or makes a chemical reaction as reflex like cuttlefish. Maybe we should delete the virus DNA information from the body, and the cell will not able to synthesize this DNA even if the cell dies. Maybe cell will destroy itself when the enemy DNA information came. I do not think that there are too many choices, and I think we can only do it during being sperm status. Even so, we may find a way to do it as after work; because for example body does not lose skin because of frictions or create some mutants after radioactivity; so it means that the body always checks the condition and this event doe not have to be by different cell's different DNAs. Maybe all DNA groups have a second order all together and some artificial which means forced by some chemical reactions for synthesis by human, plants, microorganisms or animals, cell injections change the situation. Maybe we can go from the reverse direction maybe which is the most irrelevant direction as well by right placement. Even a virus can change all the situation in theory I think and we can use radioactivity as well not for one time and calculated manner by right possible combinations. Whatever, we can easily establish some institutions on the ices with no energy requirement. We can build some long trucks for these explor- 
ers, paleontologists are included as well for universities.

We must build very wide roads even if it is city center or the other streets. We have a planet size ground and almost there is no other way to travel; but we don't have enough quality roads. We only save the current situation. We must build these roads as such big roads that it must be seen as needless like some special play grounds. We should build higher buildings instead of the smaller ones like unbreakable toys. Buildings should have gardens as much as earthquake shoes that is the base. Our vehicles should be manufactured in different sizes and we must have lighter traffic. Maybe we can build lower buildings instead of the higher ones if we use our land for agriculture efficiently; because everybody cannot sit there, also we can save materials.

Agriculture must be done as one piece by manned, unmanned air, water and land vehicles. The lands should be deposited to all the engineers and the doctors of states. They must earn their money from there, also they must give to farmers more and more than their annual earnings. Even if the work is plowing, the work should be done by the engineers or the technicians except the harvest. Maybe all of the lands should be seized by states and any people can take any agricultural products at any amount except trade. This is the best I think. We can feed the water by some electric field ion factories on the pools that I told about it the above. In this situation, the harvest is done by experts as well. There is a problem; because even if nobody needs to work to live, they need money. Maybe the old land owner farmers should use the productions to make some sauce, pickle, juice etc. with all the small town in their small towns by some grants. They can also establish any type of some animal farm maybe mixed, by grants but in their small towns, and all together. If you can feed the system to the mouth by mines, animals, foods, production etc. money will be automatically worthless. If you give to one poor person trillion dollars, the person only can make the properties be slipped to somewhere. They can't play with prices oneself and have to sell, allow to use. It does not mean nobody can use their own earned money. As always and better manner, they can use more effectively their own monies just the reverse manner if you compare the situation with the current situation.

Livestock must be developed. Farm animals can be multiplied if they can live in the nature if you let them and give time. We must multiply them by our own hands. All foods included meat can be stocked in cold storage without energy need in the required environment. We can built cold storage everywhere without energy need and by the required simple methods that I told about some of them.

Space tourism will be more important in our age. As it can be built Mars and the Moon Hotels, it will be possible to go to the Moon in a few hours and to Mars in a few days manned manner. Electronic and mechanic systems that will be used for this aim can be produced with or without magnets by many different techniques, and work forever by onetime costs. Be careful about porn stars. "Adult film" is plastering of the shit; because adults cannot watch as well. Also, it is not problem for children to find a way to watch porn. It's completely nominal. This is a government issue; because it is against philosophy of governance. People are always ignorant; but not idiot, and states educate them by schools and teachers. If they do not do this, they work on crime. They must protect any child and adult their children and they are included as well. "Nobody's business" is a sentence that everybody who is justified or unjustified can say easily and it is completely relative. People are dying of hunger, and we have not done our own businesses about space completely, and also porn is hyper immorality that no more than that. Incest, gays, lesbians or any type of porn are a serious immorality problem, and it is not less dangerous than a chemical bomb. They have to be killed as well in theory; because special life is hidden but if they do these immoralities uncovered manner then its color is going to change absolutely. Nobody wants their children that they think sex about their mothers or anyone's wife. They addict, and return is very hard. Devil is not an easy enemy. Yes maybe life still continues; but it cannot continue by porn, not so much. Porn has to be missing as well. Porn stars cannot use the boon of the technology about space. We have to have an idea honor about this subject. Nobody can condemn jealous of their families. Nobody have to be waking about porn to protect their family because of some sybarite immoral. Even its lawsuit is not possible as well. Anybody can experience sex in space with their own legal partners.

We must build for the elderly people as well. They can be controlled by a cryptic wristband like watches from a screen by self-running air vehicles that have camera, speaker and microphone. We can provide 7/24 hot and cold water for them. We must build some personal prefabricated buildings which were distributed in a huge area which is rich in the point of woods. We cannot demand money. Also we must build some free space or the Moon hospitals against some illnesses for more efficient treatments. Additionally, we must use the organs of some dead pest people legally without asking. In the concept of religion, there are some kill orders for people disrupts order. They are damned by God, angels, prophets, holy books and people, and they go to the hell forever as we can see it in

They wish to get out from the fire; but they are not the people who will get out from there. There is a constant pain for them.

Quran / Al-Ma'idah - 37

and

They are the people who buy the world against the afterlife. Their pains shall not be lightened, and they shall not be helped.

\section{Quran / Al Baqara - 86}

verses as corpses. If they are the parasites who use our possibilities irregularly and do not give some benefits then also we can use their organs legally to make some people and families happy. They are not valuable as much as a drop of tear of a mother, father or child.

Penalty of the people fighting against God and his messenger, and the people who work for breaking the order is their killing, hanging or cutting of their hands and feet crosswise or exile from the place where they are.

Quran / Al-Ma'idah - 33

and

For the Lord watches over the way of the righteous, but the way of the wicked leads to destruction.

Psalm 1:6

verses talk about kill order, and there are many kill verses in holy books. There are so many people who are waiting for organs. Their families pull the curtain to their life because of some diseases like solar eclipse. We must make them happy by these organs. I hate the people who talk about ethic in a demonic state of mind, mood and mercy for some subjects; because they always leave a worse legacy for the next, and 
save the day. The people who are in these states of mind is the hedonistic according to me. This is not a virtue. Terrorists, serial killers, rapists, and gangs, not all the people who were sentenced to death by justice; but like these ones have to be killed, and then we must use their organs. They are the danger for us, anybody of us. Showing mercy for them will be heartlessness for innocent people, order people. We have to show mercy about doctors, that I mean our justice people. Doctors always take care about our every crap for us. By the way, beside elderly people also we must develop some powerful body skeletons for people who have a defect in some part of the body

\subsection{Environment}

Some ionizer air vehicles must be produced against super volcanoes and forest fires. For forest fires, it can be used some reactions which use oxygen from air, and in a time interval damp the fire; but these ionizer air vehicles can also be used for volcano or asteroid ashes by help of all of the world. They must be exist every state. Also maybe strong shocks can be used by using air or by using heated liquid air for some strong continuous blows or maybe liquid air (Nitrogen for diffusion explosion) itself directly instead of water. Also ground itself can be used as powder with a digger by self-running generators for forests and maybe for buildings by hydraulic arms without time loss. If energy is not a problem, nothing is problem.

To combat storms, it must be built huge pumps on oceans; so we can set the heat of water as we know a reason of storms is oceans. These platforms can be built much; thus they can be used as shelter.

Air, water and land lives can be followed, and these displays can be recorded by the method stated the above even if they are watched or not. A center can be built and provides unlimited record. Animals can be followed by unmanned air vehicles that produce their own energies and silent, protected against EM waves, maybe autonomous or remote controlled by some fixed non-orbital cheap small satellites.

\subsection{Technology}

We have only one world and there have not been yet some buildings like computing center of the earth or fixed free space radio telescope of the earth. We must press a 500 billion dollars money need quite year after year. When it was done, money would come from all the people of the earth. If all the scientists demand it especially in the family of artiodactyla that means the most efficient, helpful and biggest ones then it has to be pressed; because they are the owners of economy in the name of people and they are the court about this subject. Even its lawsuit is not possible. This does not affect the economy.

When the money pressed as the first work, we must built a computing center of the earth that works at $10^{35-40}$ Hertz by the methods stated the above. Each university in the world can use from the center about $10^{15-20}$ Hertz. For example medical doctors can work on virtual body for complications on their beds, and can share the information as an article. It will be possible to be connected to the system by a simple laptop for Doctors. The center can be used for physics, medicine, chemistry, material engineering, weather forecast, earthquakes and more. Priority is determined according to necrosis of the mankind by democratic votes. For example cancer, ALS, viruses, DNA research for Jurassic Park or new teethes for any age. The center should be built as an artificial island on Marmara Sea near Istanbul in Turkey. Quantum computers are under development, and they are very sensitive. Even so, we can build quantum computers instead of the explained ones as well. If we can simulate the universe completely, we can know how the current weather of another planet in any galaxy is; thus we can know where the habitable planets are as well. Maybe there are some plants, microorganisms or animals even if there are no other people in the universe.

And we did not create the sky and the earth and what is between them in vain. That is the opinion of the unbelievers. Woe to the unbelievers because of the fire.

Quran / Sad - 27

verse talks about it, and we must be careful about different creation creatures.

As the second work, we must built a fixed free space non orbital flat radio telescope, or maybe a huge camera or maybe both of them by some lines or maybe by many small classic radio telescopes. It must be big like city centers, cities and maybe countries year after year. New parts of the telescope fill one by one circumference of the telescope and when a radius is completed then the telescope can continue the work with its new size by a single push button, and in this way the telescope can be constructed year after year in a desired size. The data can be easily processed by processors which I told the above in the article.

Word processors must be developed. In theory, each article will be written about any subject exists in our languages; because they occur by our words. We can't know them completely even if we use the processors; but we must program some artificial intelligence no matter what the cost by using eliminations. Even a laptop is enough for calculation and program area. For translated texts, essential condition is confirmation of the translators that graduated from the university. Also a center can provide some possibilities about sharing articles, and all the journals use the same center to save articles against plagiarized materials as absolute control is actually not possible by journal editors Also authors can use the system to check the condition before the submission. These word processors are needed especially for scientific articles. Every nation must protect their language, national dresses and all other cultural heritage. One type of suits and global language are a nonsense. Earning money is not problem. The main work is protect our own culture. Any works of the world must follow us; but we do not have to follow the works. The fondness must be for our honor. Their own smoke must smoke top of the each nation.

Also one of his evidences is his creation of skies and the earth, differences between your languages and colors. Surely, there are evidences for the people who knows and understands about it.

Quran/ Rum -22

In Turkey, we spend nearly 3 years to learn it in primary, high school and for university. Every nation can save time. It is not so hard to find a translator for technology learning by some committees. If a state doesn't want you to learn their technologies, there is no way to learn it. Going out for education is nonsense. Even there is no the smallest benefit. Every nation should use their own brains in their own language. The brains cannot work in other states by the way. They should look for some possibilities to make their lands as wished states. There is no difference. Maybe only a few universities can provide foreign language and the rest provide optional. If the word processors are done, the rest is not important; because it will not be so hard to make some translators that work according to sound vibrations for example as glasses that know and show the word owners separately. We must bring half residential schools even for primary school as absolute education 
system; because there is no other work. All of the students should be at the same place in a city. Maybe in a few campuses for big cities like Apple Campus with the best social facilities, the best possibilities, and they can only go to home at the weekends by campuses' own free energy vehicles. In the system, we can do more practice for small sky doctors and can be social. The campuses can be built by current schools' energy costs. Long holidays, short lesson duration, short education duration, more social experiences, more effective life style and more success.

We must wear all of the streets by street cams without blind spot. All of the record for all of the world can be saved in a small area by the above stated methods. Criminal follow can be done by induction and deduction methods without any program by a huge servant army; but the same processors can scan all of the database of all the world every second the past records and still viewed are included.

Recovery of information is possible for any information type; but during the process, the information which is searched for and any information become the same at a point relatively for us; but not for the infinite as stated the above. At this point, it must be possible an absolute hardware security system. For example, a relative but exact random is possible since relativity warned because of threshold values, that the random cannot be estimated by statistics or any precision of enemy hardware. If you assume, that you use $10^{20}$ atom groups which are switched, then for the next part of the force or energy after the comma, the sum of threshold values under relatively to a system or real threshold values affect the groups by the switches, by jumping unpredictable to other group. Maybe you can calculate a few move; but then suddenly jumps to a different group, to any group. By this way, like in 6.2 we can choose the cutting rule character random and save them for both computers which are in a communication status, or express the data according a rule. The saved data may be fake and the problem is the definition of a reference pillar which is the basic reason of hacking that is possible for any system except relative uncertain systems according to me. Maybe we can do an unlimited loop that is mixed as much as possible, in an interval we extract the data by some rules. Errors that are caused by vibrations or atmosphere are included as well are positive effects. Now, maybe this can be used for the communication of two computers as one-piece by some data process methods. Maybe we can play with unknown characters that are expressed by atoms, atom groups or a high frequency processor that works for example for a Morse alphabet by a fast data storage device which all bit unites are accessible at the same time unlimited manner since the hardware is suitable, that I explained a bit cutting method in 6.2. Maybe both of the computers have the same rule for encoding in interim memories which are openable and closable to internet and to the computers by some switches. Maybe the rule will be auto-cleanable and re-writable by an artificial intelligence. Also if we bury the cut information like in 6.2 in a certain fixed long bit group for each send, viruses can be found as well in these front memories. The system's engineers cannot know what is the situation inside or what will be approximately as well; but the computers do something that is wished. Looked for and any information can be the same at a point by compressing any size data by required rules. For example long data will be compressed more and short data will be buried into a fake data group. Also we can play onto the third computer directly; because by random, watched data will not be dependent of a function for example if you assume that the data is odd numbers. We should catch the smoothing method or its opposite by roughness the same as mirrors. Maybe different methods can be developed if we think on uncertainty and random. Even if quantum computers are included for hacking, any information is the same for searched information in a gap if we can achieve. Quantum computers can solve the situation; but it does not mean anything for it or mind.

\subsection{Economy}

State is management in theory. States are discipline and service establishments which are established on thousands of years' social experiences. People run behind some various works according to their abilities. As one person is in need for work of another one, they must use their different products. If it is controlled healthy, more work directly means more buyer; because a company needs work with more company or must buy more from some companies; so directly or indirectly, they earn more. Mankind is insatiable and everything is a distraction, a time killing and a business; thus an international organ should give money to the people who want to do business, in the name of the states according to the some standards which are determined by the organ. Doctors must be included for research and development. When the some main needs such as food, energy, water, transportation and housing are absolutized freely sustainable manner no economic crisis can affect states, companies and thus people. Inflation will not be important as much as the previous situation. Even if a bankruptcy occurs in companies, a privilege can be recognized for experienced companies even if everybody will be benefited; because environment, infrastructure and experience is very important for quick, qualified and safe works. At least, people need serve. For example, a car company can declare holiday in the company even if there are many cars unsold. They are hided for a while to be sold. They do not use their own money to do business, they use the grant; but if they win the race, they can spend their own earned money. All the works will be controlled whenever possible; so if one day a frivolity is determined that I do not mean unsold cars or research and development money, big penalties will be approved without doubt. They are going to do the work as they are using their own money. All the decisions can be done law court even for the money providing establishment or the companies against relativity.

The aim for living is living rest in peace and providing possibility to oneself. Even if it is trade, it is not the main aim and is an after work; thus when they were done with the required placement, some people will incur losses because of the mines in bullion like mountains, free houses, cheaper than water gold, have been worthless food supplies, free energy and local transports etc.; but everybody should be willing about the change, and not stay back from new works. Technology and trade are completely like this. The main aim is not make some special companies rich and richer. If people do not want to work, they cannot earn money; but they can live forever. Otherwise they cannot drink even a cup of tea at the outside. They do not die but also cannot live a good life outside. Even if they work or do not work, they must give us a child who will be an expert and help us. Maybe we can give money to inactive people at small amounts. At the point we must give more money for workers like engineers, doctors etc. As the first work, states should solve the energy problem. We should use free energy for one-time cost and freely for people. As the second work, we should build some material production facilities for buildings by world class geniuses. These ionization facilities should be formed by many small the same parts, and if it is wished, we should be able to add new parts and enlarge the facility easily, and it will be ready to build at any size. The building plans should be bought for example by the United Nations and it should be accessible for any state. 
We should press money for energy, building and agriculture with dams and machines changes for states except the biggest ones. After that, we can do safely what we wish.

We must remove interest and forex. Money is the handle of prestige; but not always, not absolutely and not for everybody. It provides a right people to use other's effort; because it means that the money owners have been showed effort as much as the others. Except this, interest and forex are basically haul and as it is outside of an economic plan, it can drag all of the world in theory to an economic crisis. There is no bigger danger that a person play forex after playing an online game, and the person says "I'm payee." while smiling like devil. This is like an awakening by Chucky who has a blade on his hands at night; so it is annoying. If a person wins, another one loses or if the same person loses some one wins companies, providers etc. are included, that they do not have a right on this money. This is a big and unconcerned method that uses anybody's effort and money. There is no way to earn money without usual works. These monies should be used for provide some better possibilities to oneself by some plans by people in its time, in a not so much interval. If there was no labor then there will not be money; so forex and interest cannot be a sector, and they eat your potential of forcing for work in the other name money. For interest, all of the works of people belong to their states. More money wish is outside of them. Already, as you cannot let an enemy state to use your people as slave in the name of this enemy state even when you have been paid for the work to your people, you cannot let interest as well. They always find a way to steal money and exploit you by some hopes even if not all of them; but you cannot say that you can do but you cannot do for the other one. They do not want you to lose; because they always need you; thus you will not even be noticed. At this point, they seem as friendly; but they are not friends, even if they are our real life friends, not bad minded and not unconcerned. States are already forced to provide possibilities for their people, and they have to combat abuse; because it is a government issue if there is an abuse. A woman who walks in street with a sexy underwear cannot have a talking right about interest of men like "Not looking is in their own hands". Interest and forex are completely disgrace. As you cannot allow suicide, opposition of school, sex change, drugs, gambling etc. you cannot allow interest and forex in the name of the order. Shares are enough in the name of earnings even if it will be meaningless a little as well because companies can take enough money to work to their mouths if they can do it that they cannot find enough worker and manager, and also they will say that what they will do to use the money for only certain works and it will be controlled and they do not need so much to share. Still it will be safer as well I think.

We should use digital money. An ageless, stylish, programmable identity card can be used as bank account card with every money movements with dynamic and autochangeable account, card number for the person other credit and bank cards are included as well, driving license, voting card, education card, court results, penalties, what the person have been doing until now etc. are included. A post device shows the desired results. Nobody can see all the information. The same information should be saved in the state database against hack. It can be set on the post devices. Soldiers can check by the card the general orders from a system that is connected to chief of general staff. We need it especially for un-amenable generals. Also it can be used for army vehicle and equipment permission. Maybe a chip can be used as well for vehicles if it will not be a problem during a war by enemy. Every act must be recorded by the same system by soldier names by date. Nobody can change even if the system orders recorded wrongly. All of the weapons and ammo must be protected in different electronic composite cabinets that uses the energy generated by self-running personal, unattainable generators or batteries. If general staff orders then nobody can use nothing. Just punches if they wish. If an anomaly occurs at the same time a phone, or an attendant can be sent. It can be done for strengthen politic will against impacts as well. Some ministers and president can use the same system as an operator. We can prevent some serious loss of life, time and money.

The same electronic strong composite protection which cannot be opened when the electronic mechanism is destroyed must be used for civil weapons as well. Civil weapons cannot sold without the electronic composite protection. States give some time to the license owners, and at the end of the time if the people has not been bought the protection they must remove the license and take the weapon. If a young at the high school ages kills someone unjustly, then the young has to be killed. His father or mother who has the gun has to pay a high amount of money or they are arrested; because even if they did not want to kill someone directly, there is a neglect; so it is very important. We cannot play with human life like a game if we have some possibility like the protection.

We should remove armies. We should only use light defense units, especially soldiers that have operational high technology equipment. As we can see the above in 5, God has no the other choice in the point of creating the mind owners. If his wish is creating the mind owners, than they have to be tested. God did not create us for his revenge. Hell is after work as God has no other choice. You choose to go to hell. An absolute hostility mentality is not possible, and it brings curse. You cannot know who is the man of God and who will be, and also even you are at this time, will you be? We must always check the situation. Holy books and prophets only warn you even their single mission is not warning as they have an absolute authority. In our historical period, one of the most changed things is maps. There are no Rome, Attila, Genghis Khan, Nimrod, Nebuchadnezzar, Alexander the Great, Ottoman Empire, England, Spain or Persians and the others. The main aim for wars is to satisfy God by establishing his order; but it never was such that by wars perfect manner. We always collect revenge ambitions in our enemies' souls and hearths that the history shows it as. There were many prophets even if they were dependent of another prophet who has a book, who lived in the same time in different places, and maybe they never knew each other or for a while. At this situation it can be said that they were living at different planets for example; so if they wish to do communicating then they should prepare an army as the people will be tense and as a war may break out. We have much more better possibilities and the main aim for war can be done over internet, radio, television etc. instantly and constantly. We have more time to think. Also we do not need any raw material beds or energy or any other life elements; because we can produce them in any wise by electricity. The universe is enough for us, and there is enough material. If you do not transform your own place into a better situation, know that any place is the same.

When we established a relax order by self-running main vital structure, governorship will not be attractive. Also hard penalties are effective about running away from governorship. We have to use execution for administrators by votes of presidents of UN, and some courts can interest the events; because we cannot let the events that shake people's trust. Gaining trust is very hard mission, and this is not a web browser based game. There is no valuable person as much as this between us that they do wrong things. The holiness of administrators 
occurs by defending right at any price. People should live without any concern about war or economy. We can create a real trust community.

Many obstacles will occur while we are managing a state to be fair. Maybe as we cannot give to everybody what they want, sometimes we cannot give the rights to the owners; because some works may be done according to an uncertain reference. This is never acceptable; so going from the reverse direction should be the right of people; because we cannot wait absolute discipline where there cannot be absolute fair. Otherwise you must leave people; but this causes chaos and its end is a state again. States are discipline and service establishments which are established on thousands of years' social experiences for protecting people against chaos in the mix of should be discussed and be done works. If it is a state order, almost every order becomes the same by some required transformations. The main principles of states are working, looking for the perfect and for the perfection looking for positive changes if they are found, then easily keeping up with them, and maybe the most basic principle is following. The things to do are limited for management style. Following and protecting the current situation are the biggest work always. If states are discipline and service establishments, then the things above are the right of people on scientists and politicians. Otherwise states do laziness and they commit crime. Every person in a state is a government official even if they do not work; because everybody is responsible about their states and states are not independent of their people; there is no other thing named state. Some of the people cannot take money from their states because of to be government official; because the state uses the money instead of them for health, justice or education for a limited time until they work; so we all too near to each other, and we cannot remain indifferent than services. As remaining indifferent is a crime, if we have some better possibilities please do not forget a little help means so much things for some people. People can live with some main limited conditions without cost of energy, water, rent and more. When it is done, then I hope people do not lose their characters because of money, and growing youth do not work because of financial concerns and work on their own lovely works. Everything is like a game, and must be like a game; but not more. The more makes people tired, and kills their hearts, souls and feelings. If we think, we can find better ways to make them busy healthy.

\subsection{Hawking Corleone: The Grandfather}

S. W. Hawking who is former Lucasian Professor of Mathematics and his generation grew with poor technologies and techniques. The first pictures of Hubble were located on underwear, and it is an evidence of our despair and hunger. Also as far as I watched at the beginning of History Channel Light Speed Documentary, the situation of physicist Clifford Victor Johnson who is professor at the University of Southern California is really sad and can be assumed as a second evidence. I am afraid other scientists will fall into the same situation soon. I am calling Mr. Johnson. Please do not attempt the work by bicycle, be patient and stay away from such thoughts. Just get a short holiday. We will bring you closer to the light speed. As Hawking, he is the most unlucky man between them because of a bad illness which is called as Amyotrophic Lateral Sclerosis also known as Lou Gehrig's disease. Even if the disease has been turned his life into a prison, he has been settled many year's hash. He is a strong and capable man. He always searched for the cures of mankind by the way of science and mind, and he did not hold back from writing articles and books, giving conferences. Also he is a very popular, well-known scientist. We should present a personal space vehicle that can produce its own energy. He should be able to go to the space anytime with his some friends like Roger Penrose from Oxford. Also maybe Buzz Aldrin misses the moon. For example he should be able to go to the moon in 3 hours with the acceleration of the earth gravity like flying to an apartment roof vertical manner or he should be using the vehicle for near space travels around the earth. This gift is the right of him and his friends on us. Without doubt we need least a billion dollars to realize this mission. The money can be earned by online advertisements in a short time without putting our hands in our pockets. For example Google can prepare something like Google doodle. If an internet user clicks on Hawking doodle, the user watches an advertisement for a short time. Scientists can share the doodle information on their social media accounts or they can advertise it in their television and radio shows by some repeats. We just demand less than 1/500 of annual ad spending of the world. It can be found a lifetime sponsor for maintenance costs of the vehicle. The sponsor's name always exists on the vehicle and the costs cannot be as satellites or insomuch as jet fighters. No fuel and no heat. Just a vehicle that has an additional ambulance equipment. I think Hawking accepts the work, because people will be done something for him. Hawking's speech machine always says something in the same tone, but it does not mean as always for Hawking's condition. We don't know what the condition is inside; thus we must hurry. I think we can't see Hawking in the earth.

The same ad methods can be used for good works constantly by some companies like for example Google. Google can put a strip of charity under YouTube videos and if the users wish, they can click the strip and watch some ads. This is much better than to seek cash money. They can do shopping with their money instead of good works. The clicks are done by the users; but the ad show is done by the computer program, and then the users cannot use their computers for a short time; because they must watch the ads until the end. Also Microsoft can turn a hand to the work. The work can be done on MS Windows. Microsoft rewards the users by a dynamic windows user portrait or a dynamic can be hidden small desktop badge on the top right. The badge changes depended to number of the watched ads.

Also this method can be used by initiatives like Mars One. They can use their own surroundings to do it. Let's use the boon of the internet for good works more effectively.

\section{Acknowledgements}

I thank to Science Publishing Group for their providing us the boon of publishing, and fast, qualify checks and their interested postures.

Even so, Science Publishing Group was not there while I was thinking and preparing this article; so especially I thank to myself, never gave up on thinking for hours in every kind of thinking positions for 3 years and repeating doing the same things like a disease 7/24. Also I thank to my family for their understanding and patience that they did not chuck out me. At last even if sometimes it made me forget my face, a grumpy person, drink over a pot of black tea that did not make me hunger or thirsty almost every day, eat less, drink less; interrupt my sleep and made my weight from $65 \mathrm{~kg}$ to nearly 55 $\mathrm{kg}$ that I was 60 in middle school; almost never went out and professed this science mining like an ALS patient and never understood how did time pass, and it came up with some results. The above stated information are these results and all of them are discoveries which belong to me. I shall never claim patent right. The requester could use this information to make the universe a better place. Saying a certain information exists or not, and could or could not is a certain information. Assumption of exists or not is in the ratio of 50\%; if so in the 
ratio of $150 \%$ certain information exists, and I am under the impression that these information; however they are actually summary and there are lots of things to calculate, are certain. Even if there could be some mistakes in the calculations, the mechanics is strong. For that reason the rest is not important at least for me. These are the subjects that I have been thought about since elementary school, I guess because of the effect of Capricorn, and again because of the effect of my sign I constantly delay and approximately write about 3 years, even if I didn't believe completely in UFOs and sign characters until a near time that I started to believe in UFOs completely after my own theory, and I still believe in that they are the productions of mankind and in the planet earth; because even technology gains volume over time, still it is independent of time. For example, lightning and some sweater, friction-dependent voltage differences can raise awareness about electricity easily. And gravity may cause a characteristic as soul is a matter and space as well. Maybe small differences can cause some dream shocks or long dream by different mood when we are in the womb or just after birth as there is no concentrate, and is a pure surrender. This dreams may cause some experiences maybe by some different magnitude stresses like lived for a long time and had a character. At this situation, the light speed is still effective for external incitements as every effect can only emerge at the light speed, and also shock, sound and EM waves are after work and temporary. Gravity causes different density space that these second effects emerge on this space.

When I was young I was thinking magnets work due to their push and pull power they have, even if they are not working at that time. I thought in middle school that I can produce electricity by magnets or using particles instead of magnets because of magnetism somehow and make the most efficient imbalance engine by using free energy similar to vibration motors that in these engines, there is a circular mass which does not sit from its center to DC motor and causes vibration. I forgot them. I was believing that matter would be brittle when it is cavernous and because of the cavernous structure the only truth is mass and there is no difference between electric charge and mass, and I was believing that I could explain these simply. Matter needs previous mass always, otherwise I thought that it will be fragile and destroy itself. I was thinking that how we can imitate the universe in a small volume for energy; because there are always motions at the outside without our wills, and even if they are temporary, how we can do it in some time intervals. I knew that I will be famous and here I am, as became 28 years old, nearly $55 \mathrm{~kg}$ on the bed, and I do not know how it happened but an amateur physicist. After this these works passed us. Leave me, you go. I did what I said. I hope I raise awareness someone in somewhere out there and the absolute information which are hiding, respect awaken for the finder and admiration for the information itself when it comes up definitely come to light one day. Beside these ones, the information in the article proves YangMills' mass and energy gap [12]. Indeed Heisenberg proved it years ago by uncertainty principle, which is the basic law at the universe according to me; but as naturally science is a process, the universe has not fallen yet into its own place by the right function or functions. Maybe due to we could not look at the picture on the puzzle from a broad perspective, even Heisenberg's himself did not understand and could not be understood properly. I admire to our interpreting and using ability, though we do not even know absolutely what is there. All of our technology was built on an uncertainty and it was all along like by the reason of not having an alternative in terms of material and method in the enrichment period of our scientific accumulation. Still I hope we can see rise of the nations.

\section{References}

1. Werner Heisenberg. Zeitschrift für Physik, Über den anschaulichen Inhalt der quantentheoretischen Kinematik und Mechanik, March (1927), Volume 43, Issue 3, pp 172-198

2. Hedrik Lorentz. Zittingsverlag Akad, De relatieve beweging van de aarde en den aether, p. 74 (1892)

3. Jönsson C. Electron diffraction at multiple slits. American Journal of Physics, (1974) 4:4-11

4. A. Einstein, B. Podolsky, N. Rosen. Can Quantum-Mechanical Description of Physical Reality Be Considered Complete? Physical Review (1935) 47 (10): 777-780

5. Albert Abraham Michelson, Edward Morley. American Journal of Science, On the Relative Motion of the Earth and the Luminiferous Ether (1887), 34 (203): 333-345

6. Stephen Hawking loses Higgs boson particle bet, theguardian.com, Thursday 5 July 2012

7. Max Planck. On the Law of Distribution of Energy in the Normal Spectrum, Annalen der Physik (1901), vol. 4, p. $553 \mathrm{ff}$

8. Stephen W. Hawking. A Brief History of Time: From the Big Bang to Black Holes, Bantam books, p. 139. (1988)

9. Muhyiddin ibn Arabi. El-Futuhatu'l-Mekkiye, 1/2, 2/232, 4/211

10. Charles Darwin. The Origin of Species, John Murray, London (1859)

11. Goldbach, C., Letter to L. Euler, June 7, 1742.

12. C. N. Yang, R. L. Mills. Phys. Rev. 96 Conservation of isotopic spin and isotopic gauge invariance, (1954), 191-195

13. Kavak M. 2018. Complement Inferences on Theoretical Physics and Mathematics. Open Science Framework. Available online: osf.io/tw52w

There were some mistakes in the language. Sorry about this. I corrected them as much as possible. Also I discovered new physical information which contradict some of the above stated information. I published them in a new paper [13]. Also the above stated mathematical findings are not true completely. There are some serious logic voids to explain them clearly. Also for this reason, I developed new methods back to the drawing board [13].

Note on June 2018 